\title{
lin-25, a gene required for vulval induction in Caenorhabditis elegans
}

\author{
Simon Tuck ${ }^{1,2,4}$ and Iva Greenwald ${ }^{1,2,3}$ \\ ${ }^{1}$ Department of Molecular Biology, Princeton University, Princeton, New Jersey 08544 USA; ${ }^{2}$ Department of Biochemistry \\ and Molecular Biophysics, ${ }^{3}$ Howard Hughes Medical Institute, Columbia University College of Physicians and Surgeons, \\ New York, New York 10032 USA
}

\begin{abstract}
During vulval development in the Caenorhabditis elegans hermaphrodite, the fates of six vulval precursor cells (VPCs) are influenced by distinct cell signaling events. In one event, a somatic gonadal cell, the anchor cell, induces the three nearest VPCs to adopt vulval cell fates. In another event, lateral signaling between adjacent VPCs specifies one of two different vulval fates, $1^{\circ}$ and $2^{\circ}$. Induction of vulval fates by the anchor cell is mediated by a signal transduction pathway involving let-60 Ras, lin-45 Raf, and mpk-1/sur-1 MAP kinase, whereas lateral signaling is mediated by lin-12. We have shown that the mutant phenotype of lin-25, a gene required for VPC fate specification, results from a defect in vulval induction. Genetic epistasis experiments indicate that lin-25 is required in the inductive signaling pathway downstream of let-60 Ras and the Raf/MAP kinase cascade. A decrease in induction also appears to decrease lateral signaling. We have cloned and sequenced the lin-25 gene and shown that it encodes a novel protein that may be a target of the mpk-1/sur-1 MAPK.
\end{abstract}

[Key Words: Cell signaling; C. elegans; vulval development; lin-25; ras; lin-1]

Received October 3, 1994; revised version accepted December 20, 1994.

Signaling between cells is required for a variety of developmental processes including cell-fate determination, cell migration, cell proliferation, and cell shape changes. In recent years, it has become apparent that many of the molecules that mediate signaling have been very highly conserved during evolution. In many different signal transduction pathways, binding of a ligand to a tyrosine kinase type receptor leads to the sequential activation of a group of proteins: Ras, Raf, MAP kinase kinase (MEK), and MAP kinase (MAPK). Biochemical studies have shown that these proteins can direct cell proliferation or differentiation of cultured mammalian cells (for review, see Khosravi and Der 1994). Genetic analysis has shown that the same cascade mediates many different signaling events in both unicellular and multicellular organisms including the response to mating pheromones in Schizosaccharomyces pombe (Fukui et al. 1986; for review, see Kurjan 1993), growth control in Saccharomyces cerevisiae (for review, see Broach and Deschenes 1990), the induction of vulval cell fates in Caenorhabditis elegans (Beitel et al. 1990; Han and Sternberg 1990; Han et al. 1990|, the establishment of dorsoventral polarity and development of the terminal structures in the Drosophila embryo (Ambrosio et al. 1989; Brand and Perrimon 1994), and the determination of photoreceptor cell fate in the eye imaginal disc (Simon et al. 1991).

A great deal of progress has been made recently in

${ }^{4}$ Corresponding author. elucidating the events leading up to the activation of Ras by upstream components (for review, see Khosravi and Der 1994). In contrast, much less is known at the present time about the events taking place at the end of the cascade, downstream of MAPKs. Many changes induced by activation of the Ras/MAPK pathway undoubtedly result from changes in gene expression. Precisely how these come about, however, is not well understood. Furthermore, it is not known whether changes in transcription are sufficient to mediate the effects of the Ras pathway or whether changes brought about independently of immediate effects on gene expression are also important.

Work on other organisms has shown that MAPKs mediate their effects at least in part by phosphorylating transcription factors. For example in both mammalian cells and Drosophila, MAPKs are able to modulate the activities of some members of the Ets family of DNAbinding proteins (Gille et al. 1992; Marais et al. 1993; Brunner et al. 1994b; O'Neill et al. 1994). However, several other substrates have been identified for MAPK, some of which are themselves kinases (Ray and Sturgill 1987; Sturgill et al. 1988; Chou et al. 1992; Stokoe et al. 1992), suggesting that changes in gene expression might also result from the indirect activation of other factors. For example, activation of Ras in PC12 cells leads to the rapid stimulation of the CREB kinase which, in turn, activates the DNA-binding protein, CREB, which is necessary for full induction of the immediate early gene, c-fos (Ginty et al. 1994). 
In yeast, some changes induced by MAPKs occur independently of immediate changes in transcription. For example, in S. cerevisiae, the MAPK, FUS3, has at least two separate functions. One is to activate the transcription factor STE12 (Song et al. 1991), which is required for the transcription of genes in response to mating pheromones, and another is to activate the product of the FARl gene (Peter et al. 1993), a cyclin-dependent kinase inhibitor, which is required for growth arrest in the $G_{1}$ phase of the cell cycle (Chang and Herskowitz 1992).

During development of the vulva in the $C$. elegans hermaphrodite, a signal from a somatic gonadal cell, the anchor cell, causes three nearby hypodermal cells, P5.p, P6.p, and P7.p, to adopt vulval fates (Sulston and White 1980; Kimble 1981). These three cells are part of a group of six cells, P3.p-P8.p, that are collectively termed vulva precursor cells (VPCs). The VPCs are initially developmentally equivalent: Each has the potential to adopt vulval or hypodermal fates (Sulston and White 1980; Sternberg and Horvitz 1986). Classical and molecular genetic analyses have shown that the anchor cell signal, encoded by the lin-3 gene (Hill and Sternberg 1992), promotes vulval induction by activating a signal transduction pathway involving Let-23, a receptor-type tyrosine kinase (Aroian et al. 1990), Sem-5, an SH2- and SH3-domain containing protein (Clark et al. 1992), Let-60 (Ras), and Lin-45 (Raf) proteins (Han and Sternberg 1990; Han et al. 1993), and a MAPK, Mpk-1/Sur-1 (Lackner et al. 1994; Wu and Han 1994).

Precisely how the activation of $m p k-1 /$ sur-1 MAPK causes cells to adopt vulval fates is not well understood. Two genes, lin-1 and lin-31, have been shown to function downstream of $m p k-1 /$ sur-1 in the genetic pathway for VPC fate specification (Lackner et al. 1994; Wu and Han 1994), but it is not yet clear how they act at a molecular level. $\operatorname{lin}-1$ is a negative regulator of vulval induction (Horvitz and Sulston 1980; Ferguson and Horvitz 1985). Loss-of-function mutations in lin-1 cause cells that normally adopt a hypodermal fate, P3.p, P4.p, and P8.p, to instead adopt vulval fates (Horvitz and Sulston 1980; Sulston and Horvitz 1981; Ferguson et al. 1987). lin-31, which encodes a transcription factor of the HNF3/forkhead family, is involved in both positive and negative regulation (Miller et al. 1993). Mutations in lin-31 lead to defects in fate specification in all six cells, P3.p-P8.p (Ferguson et al. 1987), and the Lin-31 protein is thought to function by regulating the activities of other proteins, both positively acting determinants and negatively acting ones (Miller et al. 1993). However, until this study, no strictly positively acting factors acting at this step in vulval induction had been identified.

In this paper we describe the genetic and molecular genetic characterization of the lin-25 gene, which is required for VPC fate specification. We have shown by a combination of laser ablation experiments and genetic epistasis tests that $l i n-25$ is a positive regulator of vulval induction and that it functions downstream of let -60 ras in the genetic pathway for induction. We have cloned and sequenced the gene and shown that it encodes a novel protein that may be a substrate for the Mpk-1/ Sur-1 MAPK.

\section{Results \\ Background}

The C. elegans vulva, the structure linking the uterus to the exterior, is generated from the 22 descendants of 3 ventral hypodermal cells, P5.p, P6.p, and P7.p (Sulston 1976; Sulston and Horvitz 1977). These three cells are part of a group of six cells, P3.p-P8.p, that are initially developmentally equivalent (Fig. 1) (Sulston and White 1980). The fates of these six cells, termed collectively the vulval precursor cells (VPCs), are influenced by two or possibly three distinct cell signaling events (Kimble 1981; Sternberg and Horvitz 1986, 1989; Herman and Hedgecock 1990). The anchor cell is thought to induce the three VPCs closest to it, P5.p, P6.p, and P7.p, to adopt vulval fates (Sulston and White 1980; Kimble 1981) (Fig. 1). Two different vulval fates are seen in wild-type worms, $1^{\circ}$ and $2^{\circ}$. P6.p, which lies closest to the anchor

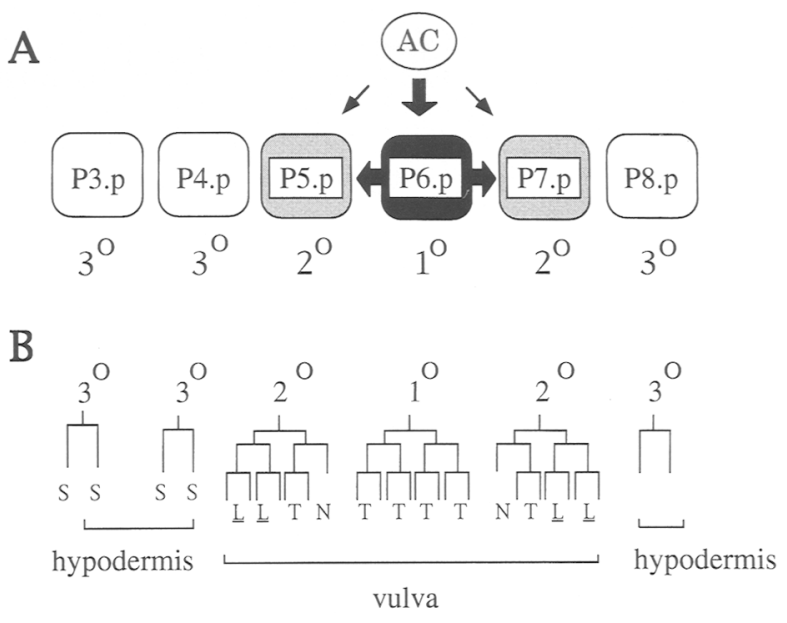

Figure 1. (A) A model for signaling in the developing vulva (based on Horvitz and Sternberg 1991). A gonadal cell, the anchor cell $(\mathrm{AC})$, induces nearby cells to adopt vulval cell fates (Kimble 1981). VPCs induced to adopt a vulval fate signal laterally to one another causing P5.p and P7.p to adopt the $2^{\circ}$ fate (Sternberg 1988). The precise order of the signaling events is not yet known. The putative inhibitory signal between hyp7 and the VPCs (Herman and Hedgecock 1990) is not shown. (B) Each fate is to give rise to a lineage, that is to undergo a particular pattern of cell divisions and to generate a characteristic set of cell types. For the VPCs, the pattern formed by the planes of cell division and the adhesion properties of the granddaughters is a useful marker for the VPC fates (Sternberg and Horvitz 1986; see Table 2 legend). These planes are designated $T$ or $L:(T)$ a transverse (left-right) division (with respect to the symmetry of the animal); (L) a longitudinal division (anterior-posterior); (N) the cell does not divide. If the cells produced by a longitudinal division adhere to the ventral cuticle this is denoted by underlining (i.e., L). P5.p and P7.p generate mirror symmetric lineages both of which are considered $2^{\circ}$. (S) The cell fuses with the large hypodermal syncytium, hyp7, which surrounds the VPCs. 
cell, adopts the $1^{\circ}$ fate, whereas P5.p and P7.p adopt the $2^{\circ}$ fate. The choice between $1^{\circ}$ and $2^{\circ}$ fates is thought to be controlled by a second signaling event in which VPCs signal laterally to one another (Sternberg 1988). In wildtype hermaphrodites P3.p, P4.p, and P8.p do not give rise to cells contributing to the vulva but, instead, generate hypodermal cells [the hypodermal $\left(3^{\circ}\right)$ fate]. There is evidence that a large syncytial cell, hyp7, which surrounds the VPCs participates in a third signaling event that inhibits the VPCs from adopting vulval fates (Herman and Hedgecock 1990).

Mutations affecting vulval development are of two types, vulvaless and multivulva (Horvitz and Sulston 1980; Sulston and Horvitz 1981; Ferguson and Horvitz 1985). Vulvaless mutations prevent P5.p, P6.p, and P7.p from adopting vulval fates: Animals carrying such mutations have no vulva and are therefore unable to lay eggs. Multivulva mutations cause the opposite phenotype. In animals carrying these mutations, cells that normally adopt the hypodermal fate, P3.p, P4.p, and P8.p, instead adopt a vulval fate $\left(1^{\circ}\right.$ or $\left.2^{\circ}\right)$ (Ferguson et al. 1987). Multivulva mutations, therefore, cause vulval tissue to be generated at ectopic sites. The pseudovulvae so formed are clearly visible under the dissecting microscope as protrusions from the ventral hypodermis (Horvitz and Sulston 1980; Sulston and Horvitz 1981).

\section{The lin-25 null phenotype}

Ten lin-25 alleles are presently known. lin-25(e1446) and lin-25(n545ts) were found in genetic screens for mutations affecting egg-laying (Trent et al. 1983; Ferguson and Horvitz 1985). Two additional alleles, $\operatorname{ar} 90$ and n1063, were isolated in noncomplementation screens that made no assumptions about the nature of the null phenotype (see Materials and methods; K. Edwards and H.R. Horvitz, pers. comm.|, and six other alleles were isolated in a number of other genetic screens (S. Clark and H.R. Horvitz; H. Chamberlin and P. Sternberg; D. Eisenmann and S. Kim; M. Han; all pers. comm.). The phenotypes conferred by the seven strongest alleles, e1446, ar90, n1063, ga67, ku70, ku77, and ku78, are indistinguishable (Table 1A). Approximately $6 \%$ of hermaphrodites homozygous for any of these alleles die as larvae, a slightly greater proportion are sterile, and the remainder (the majority) are fertile but completely egglaying defective. All 10 lin-25 mutant alleles are recessive to wild type. Furthermore, animals homozygous for $n 1063$ but bearing a duplication of the region are able to lay eggs (Table 1B).

The seven strong lin-25 mutations appear to be null alleles by the following criteria. First, for all seven alleles neither the larval lethality nor the sterility defects are markedly enhanced in trans to a deficiency of the region, arDf1 (Table 1B; data not shown). Second, lin-25(n1063) behaves like a deficiency in trans to $\operatorname{lin}-25$ (n545ts), at $20^{\circ} \mathrm{C}$. At this temperature, $\operatorname{lin}-25$ (n545ts) is a hypomorphic (reduced activity) allele (Table 1B). Third, lin25 (ar90) is suppressed by the amber suppressor tRNA encoded by sup-7(st5) (Waterston 1981; Wills et al. 1983) (see Materials and methods). Finally, we have sequenced lin-25(ar90) and found that the mutation creates an amber codon that would lead to a severely truncated protein (see below).

Ferguson et al. (1987) have described three different effects of lin-25 mutations on postembryonic development. First, during the L1 stage, in $\sim 75 \%$ of the animals, one of the six cells, P3.p-P8.p, divides to produce two daughters that subsequently both become VPCs. lin-25 mutant hermaphrodites, therefore, often contain seven VPCs instead of the normal six. Second, during the L3 stage, lin-25 mutant animals show defects in VPC fate specification. Third, temperature-shift experiments indicate that lin-25 is required for the development of some component of the egg-laying apparatus during the early L4 stage. In this paper we have focused on the role of lin-25 in VPC fate determination. Some additional observations on other defects are described in Materials and methods.

In lin-25(e1446) mutant hermaphrodites P5.p and P7.p, which normally adopt the $2^{\circ}$ fate, instead adopt the $3^{\circ}$ fate (Ferguson et al. 1987). In many animals P6.p adopts the $1^{\circ}$ fate but in others the fate adopted by P6.p does not correspond to any seen in wild-type animals. We have analyzed the VPC lineages in animals carrying a different allele, lin-25(n1063), (Table 2) and found a similar pattern. In these animals P5.p and P7.p undergo the $3^{\circ}$ lineage and P6.p often generates a hybrid lineage, which either has characteristics of two different vulval lineages $\left(1^{\circ}\right.$ and $\left.2^{\circ}\right)$ or of a vulval lineage and the nonvulval $\left(3^{\circ}\right)$ one.

lin-25 is not required for specification of the $2^{\circ}$ fate if Lin-12 is activated

The absence of cells expressing the $2^{\circ}$ fate could arise for two quite different reasons. First, it could result from a defect in lateral specification. For example, mutations affecting the production of the lateral signal or its reception or transduction in P5.p and P7.p might result in P5.p and P7.p failing to adopt the $2^{\circ}$ fate. Alternatively, it might indicate a defect in vulval induction. In hermaphrodites carrying certain hypomorphic mutations in 1 in-3, let-60, and sem-5, genes encoding components of the inductive signaling pathway, the pattern of fates adopted by the VPCs is similar to that seen in lin-25 mutants (Sulston and Horvitz 1981; Beitel et al. 1990; Clark et al. 1992).

To investigate the possibility that lin-25 might be required for lateral signaling we constructed $\operatorname{lin}-12(d) /+$; lin-25(0) double-mutant animals. $\operatorname{lin}-12$ is thought to be the receptor for the lateral signal. Dominant, hypermorphic mutations in $l i n-12$, such as lin-12(n137), cause all six VPCs to adopt the $2^{\circ}$ fate, and hermaphrodites carrying such mutations are Multivulva (Greenwald et al. 1983). Iin-25(e1446) had no effect on the Multivulva phenotype of $\operatorname{lin}-12(n 137) /+$. One hundred percent $(215 /$ 215) of the 1 in-12(n137)/ + ; lin-25(e1446) double-mutant 
Table 1. lin-25 alleles and gene dosage studies with lin-25

\begin{tabular}{|c|c|c|c|}
\hline \multirow[b]{2}{*}{ Allele ${ }^{a}$} & \multicolumn{3}{|c|}{$\begin{array}{r}\text { A. Comparison of lin-25 alleles } \\
\text { Percent }\end{array}$} \\
\hline & $\mathrm{Egl}^{\mathrm{b}}$ & sterile $^{\mathrm{b}}$ & $\begin{array}{l}\text { larval } \\
\text { arrest }^{\mathrm{c}}\end{array}$ \\
\hline$e 1446$ & $100(231 / 231)$ & $10(28 / 278)$ & $7(19 / 278)$ \\
\hline $\operatorname{ar} 90$ & $100(273 / 273)$ & $14(48 / 337)$ & $5(16 / 337)$ \\
\hline n1063 & $100(159 / 159)$ & $14(28 / 199)$ & $6(12 / 199)$ \\
\hline$k u 78$ & $100(172 / 172)$ & $12(25 / 211)$ & $7(14 / 211)$ \\
\hline ku 70 & 100 (many) & - & - \\
\hline ku77 & $100(156 / 156)$ & $12(23 / 191)$ & $6(12 / 191)$ \\
\hline ga67 & $100(287 / 287)$ & $9(30 / 342)$ & $7(25 / 342)$ \\
\hline sy29 & $100(374 / 374)$ & $5(18 / 393)$ & $<1 \quad(1 / 393\}$ \\
\hline$n 545 t s$ & $67(170 / 255)$ & $0 \quad(0 / 255)$ & $<1 \quad(1 / 255)$ \\
\hline \multirow[t]{2}{*}{$n 1722 t s$} & $2 \quad \mid 5 / 246\}$ & $0 \quad(0 / 246)$ & $0 \quad\{0 / 246\}$ \\
\hline & \multicolumn{3}{|c|}{$\begin{array}{r}\text { B. Gene dosage studies with lin-25 } \\
\text { Percent }\end{array}$} \\
\hline Genotype & $\mathrm{Egl}^{\mathrm{b}}$ & sterile $^{b}$ & $\begin{array}{l}\text { larval } \\
\text { arrest }^{\mathrm{c}}\end{array}$ \\
\hline$\overline{n 1063 / a r D f 1^{\mathrm{b}, \mathrm{d}}}$ & $100(167 / 167)$ & $20(46 / 224)$ & $5(11 / 224)$ \\
\hline$n 1063 /+$ & $0 \quad(0 / 185)$ & $0(0 / 185)$ & $0 \quad(0 / 185)$ \\
\hline n1063/n1063/+c & $4 \quad(6 / 168)$ & $2(3 / 171)$ & $0 \quad \mid 0 / 171)$ \\
\hline$+1+1+c$ & $3 \quad(7 / 212)$ & $2(5 / 217)$ & $0 \quad(0 / 217)$ \\
\hline$n 545 t s / n 1063^{f}$ & $85(231 / 271)$ & $0 \quad(0 / 271)$ & $0 \quad(0 / 271)$ \\
\hline$n 545 t s / a r D f 1^{f}$ & $89(193 / 217)$ & $0(0 / 2.17)$ & $0 \quad|0 / 217|$ \\
\hline
\end{tabular}

${ }^{a}$ All alleles are EMS induced. The isolation of $e 1446$ and n545ts has been described elsewhere (Ferguson and Horvitz 1985). The isolation of $a r 90$ is described in Materials and methods. $n 1063$ and n1722ts were obtained from K. Edwards, S. Clark, N. Young, and H.R. Horvitz (MIT, Boston, MA). ku70, ku77, and ku78 were obtained from M. Han (University of Colorado, Boulder). ga67 was obtained from D. Eisenmann and S. Kim (Stanford University, CA). sy29 was obtained from H. Chamberlin and P. Sternberg (Caltech, Pasadena, CA). The complete genotype of $k u 70$ was dpy-11(e224) lin-25(ku70).

${ }^{\text {b}}$ The complete broods of 5-8 hermaphrodites were scored first for larval lethality. Animals that survived were then scored for fertility and for the ability to lay eggs. (Egl) Egg-laying defective. Percentages of Egl animals given are the percentages of surviving and fertile animals that were Egl rather than of the whole population. Animals were scored as sterile only if they had no progeny. Many other animals had much reduced brood sizes. Animals homozygous for $e 1446$, ar90, n1063, ku70, ku77, ku78, and ga67 are completely egg-laying defective providing their parent was also homozygous; partial maternal rescue is seen when the parent is heterozygous (see Materials and methods).

${ }^{c}$ It is not known why some lin-25 animals die as larvae (see Materials and methods).

dComplete genotype unc-42(e270) arDf1/sma-1(e30) lin-25/n1063). arDf1 deficiency homozygotes die before hatching (Ahnn and Fire 1994; R. Barstead, pers. comm.). Unhatched eggs were therefore assumed to be of the genotype arDf1/arDf1 rather than arDf1/lin25(n1063). The Sma phenotype of sma-1(e30) is easily scored in newly hatched $\mathrm{L}_{1}$ larvae so that it was possible to distinguish dead larvae of the genotype $\mathrm{lin}-25(\mathrm{n} 1063) / \mathrm{lin}-25(\mathrm{n} 1063)$ from those of the genotype $\mathrm{in}-25(\mathrm{n} 1063) / \operatorname{arDf} 1$. The proportion of Sma to non-Sma animals in the population surviving to become adults was not significantly different from the expected ratio of $1: 3$. Furthermore, the proportion of unhatched eggs to hatched was $1 / 4$. These results suggest that the large majority of $\operatorname{lin}-25(\mathrm{n} 1063) /$ arD 1 animals hatched. ${ }^{\text {c} C o m p l e t e ~ g e n o t y p e s ~ s m a-1(e 30) ~ l i n-25(n 1063) ; ~ c t D p 8 ~ a n d ~ u n c-42(e 270) ~ s m a-1(e 30) ; ~ c t D p 8, ~ r e s p e c t i v e l y . ~ D u p l i c a t i o n s ~ a n d ~ d e f i c i e n-~}$ cies often cause low penetrance egg-laying defects for reasons that are not understood.

${ }^{f}$ The percentages given are for trans heterozygotes arising from a cross in which one parent carried a wild-type lin-25 allele. Animals of these genotypes coming from hermaphrodites lacking a wild-type lin-25 allele are $100 \% \mathrm{Egl}$.

animals were Multivulva. We followed the lineages generated by the VPCs in eight double-mutant hermaphrodites. In most individuals, most of the VPCs adopted the $2^{\circ}$ fate (Table 2). Furthermore, in double-mutant animals carrying a molecular marker for the $2^{\circ}$ fate, $l a c Z$ under the control of lin-11 gene regulatory sequences (Freyd et al. 1990; G. Freyd and H.R. Horvitz, pers. comm.), the pattern of expression of the reporter was consistent with all cells having adopted the $2^{\circ}$ fate (data not shown). Thus lin-25 is not required for the specification of the $2^{\circ}$ fate in the presence of activated Lin- 12 .

lin-25 is required for vulval induction in the absence of lin-15 activity

We reasoned that the lin-25 mutant phenotype might be explained if the gene product were required upstream of lin-12 for the production or function of the lateral signal, or were itself the signal. To test this possibility we con- 
Table 2. Vulval fates in lin-25 mutant animals

\begin{tabular}{|c|c|c|c|c|c|c|c|c|}
\hline Genotype & $\mathrm{AC}$ & P3.p & P4.p & P5.p & P6.p & P7.p & P8.p & Pn. $p^{a}$ \\
\hline \multirow[t]{2}{*}{ Wild type $^{b}$} & + & $3^{\circ}$ & $3^{\circ}$ & $2^{\circ}$ & $1^{\circ}$ & $2^{\circ}$ & $3^{\circ}$ & \\
\hline & + & SS & SS & $\underline{\text { LLTN }}$ & TTTT & NTLL & SS & \\
\hline \multirow[t]{7}{*}{$\operatorname{lin}-25(n 1063)^{c}$} & + & $3^{\circ}$ & $3^{\circ}$ & $\overline{3^{\circ}}$ & $1 \%$ ind & $3^{\circ}-$ & $3^{\circ}$ & $3^{\circ}$ \\
\hline & + & SS & SS & SS & TTTS & SS & SS & \\
\hline & + & SS & SS & TTTT & TO S & $S$ & SS & \\
\hline & + & SS & SS & SS & OSOL & SS & SS & \\
\hline & + & SS & SS & SS & SS & STLT & SS & SS \\
\hline & + & SS & SS & SS & S SS & SS & SS & SS \\
\hline & + & SS & SS & S TO & TOLT & SS & $\mathrm{S}$ & \\
\hline \multirow[t]{5}{*}{$\operatorname{lin}-25(n 1063)$} & $-{ }^{d}$ & $3^{\circ}$ & $3^{\circ}$ & $3^{\circ}$ & $3^{\circ}$ & $3^{\circ}$ & $3^{\circ}$ & $3^{\circ}$ \\
\hline & - & $S$ & SS & SS & SS & $\mathrm{S}$ & SS & \\
\hline & - & $\mathrm{S}$ & SS & SS & SS & SS & $\mathrm{S}$ & SS \\
\hline & - & SS & SS & SS & SS & SS & SS & SS \\
\hline & - & SS & SS & SS & SS & $\mathrm{S}$ & SS & \\
\hline $\operatorname{lin}-12(n 137) /+^{e}$ & $-^{f}$ & $2^{\circ}$ & $2^{\circ}$ & $2^{\circ}$ & $2^{\circ}$ & $2^{\circ}$ & $2^{\circ}$ & \\
\hline \multirow{9}{*}{$\begin{array}{l}\text { lin-12(n137)/t } \\
\quad \operatorname{lin}-25(e 1446)\end{array}$} & $-^{\mathrm{f}}$ & $2^{\circ}$ & $2^{\circ}$ & $2^{\circ}$ & $2^{\circ}$ & $2^{\circ}$ & $2^{\circ}$ & $2^{\circ}$ \\
\hline & & LLON & $\underline{\text { LLLO }}$ & $\underline{\text { LLTN }}$ & $\underline{\text { LLTN }}$ & LLOO & NSLL & $\underline{\text { LLOO }}$ \\
\hline & & LOTT & $\overline{\mathrm{OL}} \underline{\mathrm{L}}$ & LLTN & LLTT & NOSS & LLTN & \\
\hline & & $\overline{\text { LLTO }}$ & $\underline{\text { LLTN }}$ & $\overline{\text { LLTT }}$ & $\overline{\mathrm{LO}} \underline{\mathrm{LL}}$ & NTEL & $\overline{\mathrm{LLT}}$ & $\underline{\text { LLOT }}$ \\
\hline & & $\underline{\overline{L L}} L T$ & LLON & $\overline{\underline{L L}} L L$ & $\underline{L L L N}$ & $\underline{\text { SSTN }}$ & $\overline{\text { LLTT }}$ & $\underline{\underline{\mathrm{LL}} L O}$ \\
\hline & & $\overline{\text { LLLL }}$ & LLTT & $\overline{\mathrm{OOLL}}$ & $\overline{T L} \underline{L L}$ & $\overline{L L L L}$ & NOLT & \\
\hline & & LLOT & LLTO & $\underline{L L L N}$ & LTLL & OTLL & LNLL & \\
\hline & & $\overline{\text { SS }}$ & $\underline{\mathrm{LLTT}}$ & $\overline{\mathrm{OLTT}}$ & NTLL & $\operatorname{LLL\overline {L}}$ & $\mathrm{LO} \overline{\mathrm{LL}}$ & \\
\hline & & $\underline{\text { LLTT }}$ & $\underline{\text { LLTO }}$ & $\underline{\text { LLON }}$ & $\underline{\text { LLNT }}$ & NT⿱亠䒑LL & 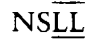 & $\underline{\text { LLOT }}$ \\
\hline
\end{tabular}

Each line showing lineages represents the vulva lineages of an individual animal. See Fig. 1 legend for the meaning of $\mathrm{S}, \mathrm{L}, \mathrm{T}$ and $\mathrm{N}$. (O) Oblique division. Consensus fates derived from several lineages are shown above each group of lineages, using the criteria of Sternberg and Horvitz (1986). $\left(3^{\circ}\right) \mathrm{S}$ and SS; $\left(2^{\circ}\right)$ LLTN, LLTT, LLTO, LLOT (or their mirror images); ( $\left.1^{\circ}\right)$ TTTT (or any lineage in which all four descendants of a VPC generate cells that fail to adhere to the ventral cuticle); (ind) cells were induced, but lineage generated was not clearly $1^{\circ}$ or $2^{\circ}$. Lineages were followed from the mid-L 3 stage onwards.

"In hermaphrodites carrying strong lin-25 mutations one of the cells, P3.p-P8.p, often divides during the LI stage to produce two cells both of which subsequently become VPCs (Ferguson et al. 1987). lin-25 mutant hermaphrodites therefore often have seven rather than six VPCs. We did not determine which cell had divided precociously in these animals: Cells are therefore numbered arbitrarily starting at P3.p, the most anterior VPC.

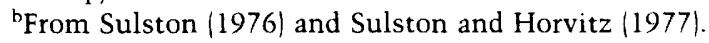

"The lineages generated in Iin-25(e1446) are described in Ferguson et al. (1987). The position of the AC with respect to the VPCs in lin-25 mutant hermaphrodites is somewhat variable.

$\mathrm{d}$ - ) Animals with no anchor cell. The anchor cell was removed by ablating the progenitors of the somatic gonad, Z1 and Z4, during the Ll stage.

'From Mango et al. (1991).

${ }^{f}$ Most lin-12(n137)/ + hermaphrodites have no anchor cells (Greenwald et al. 1983).

structed double mutant animals containing a mutation in $\operatorname{lin}-25$ and a mutation in another gene required for correct VPC fate specification, lin-15. lin-15 is a negative regulator of vulval induction (Ferguson et al. 1987; Clark et al. 1994; Huang et al. 1994). In lin-15(n309) mutant hermaphrodites, all six VPCs adopt a vulval fate even in the absence of the anchor cell (and the rest of the somatic gonad) (Ferguson et al. 1987; Sternberg and Horvitz 1989). In these animals, lateral signaling between adjacent VPCs results in a pattern in which cells adopting the $1^{\circ}$ and $2^{\circ}$ fates alternate along the ventral hypodermis (Ferguson et al. 1987; Sternberg 1988). This pattern requires lin-12 activity: In lin-12(0); lin-15(n309) doublemutant hermaphrodites, all six VPCs adopt the $1^{\circ}$ fate and the animal is Multivulva, even in the absence of the gonad (Sternberg and Horvitz 1989). If lin-25 were required specifically for lateral signaling in the vulva, the lin-25(0); lin-15(n309) double mutant should resemble the lin-12(0); lin-15(n309) double mutant. All six VPCs should adopt the $1^{\circ}$ fate, and such animals would be Multivulva. We found instead that lin-25 mutations strongly suppressed the Multivulva phenotype of lin-15(n309) (Fig. 2). The effect of lin-25 mutations on the fates adopted by the VPCs in lin-15 mutant animals was most clearly seen in the absence of the gonad. In lin-25(0); lin-15(n309) double-mutant hermaphrodites in which the gonad had been ablated most VPCs adopted the nonvulval $\left(3^{\circ}\right)$ fate, or expressed hybrid lineages, suggesting that they had been only partially induced (Table 3 ). These results suggest that lin-25 is required for induction of vulval fates rather than specifically for lateral specification.

It was not possible to examine the efficiency of lateral signaling in lin-25(0); lin-15(n309) double-mutant her- 
Figure 2. Phenotypes of adult hermaphrodites carrying mutations affecting vulval development. All animals are viewed by Nomarski differential interfence contrast microscopy. (N2) The wild-type strain. The arrowhead indicates the position of the vulva. (lin-25) Complete genotype lin25(e1446). This animal has a small protrusion at the vulva characteristic of lin-25 mutant hermaphrodites (Ferguson and Horvitz 1985). (lin-12(d)/ + Complete genotype dpy-19(e1259)lin-12(n137)/unc32(e189). lin-12(d)/ + hermaphrodites are Multivulva (Greenwald et al. 1983). Ectopic pseudovulvae are marked with arrowheads. $\operatorname{lin}-12(d) /+$; lin-25(0), complete genotype $d p y-19(e 1259) l i n$ 12(n137)/unc-32(e189): lin-25(e1446). lin25 does not affect the Multivulva phenotype caused by $\operatorname{lin}-12(d) /+$. (Iin15(0)) Complete genotype lin-15(n309). lin-15 mutant animals display a Multivulva phenotype distinct from that shown by lin-12(d) mutant animals (Ferguson and Horvitz 1985; Ferguson et al. 1987). The pseudovulvae are larger and less regularly spaced. (lin-25(0); lin-15(0)) Complete genotype lin-25(e1446); lin-15(n309). lin-25 mutations suppress the Multivulva phenotype caused by lin-15 mutations. (let 60(gf) ) Complete genotype let-60(n1046). The Multivulva phenotype caused by let $60(g f)$ is similar to that caused by lin-15 mutations (Ferguson et al. 1987; Han et al. 1990). (let-60(gf); lin-25(0)) Complete genotype let-60(n1046); lin-25(e1446). lin-25 mutations suppress the Multivulva phenotype caused by let-60(gf). $\operatorname{lin}-1(e 1777)$ is a strong reduction-of-function mutation (Ferguson and Horvitz 1985). The Multivulva phenotype is suppressed by muta-
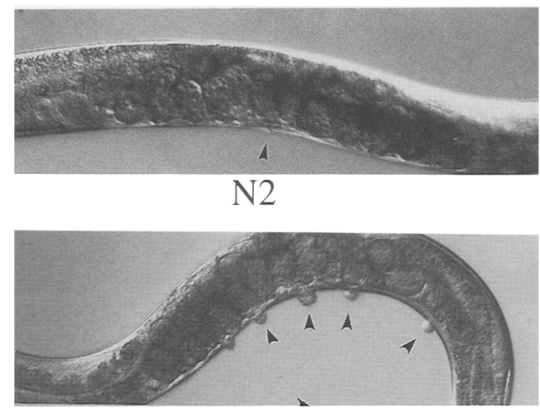

$\operatorname{lin}-12(d) /+$

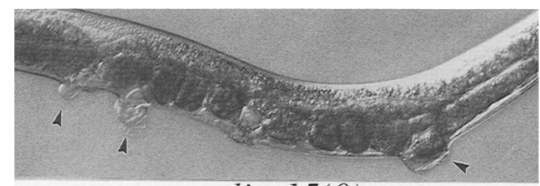

lin-15(0)
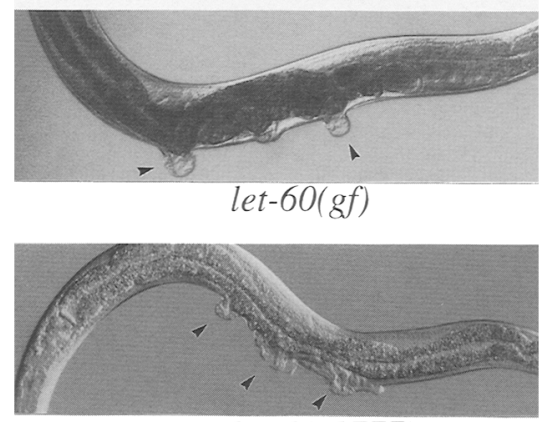

$\operatorname{lin}-1(e 1777)$

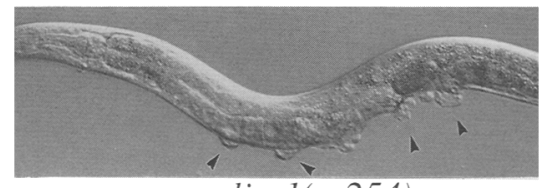

lin-1(sy254)

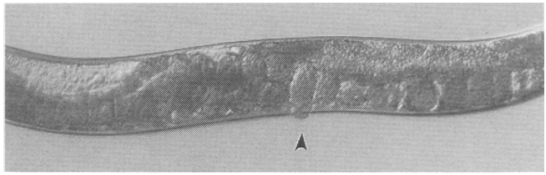

lin-25(e1446)

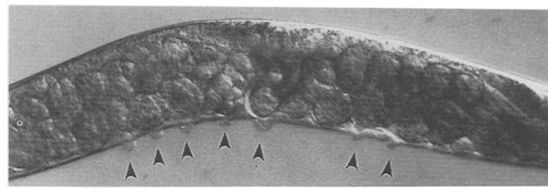

$\operatorname{lin}-12(d) /+; \operatorname{lin}-25(0)$

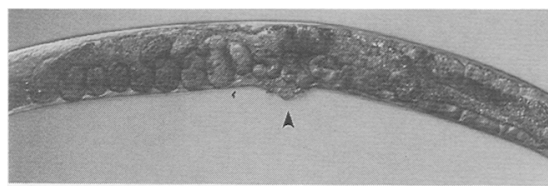

$\operatorname{lin}-25(0) ; \operatorname{lin}-15(0)$

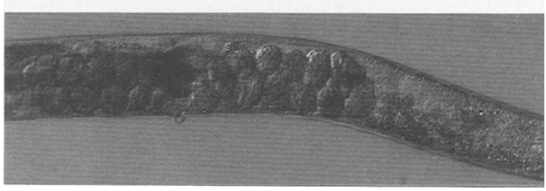

let-60(gf); lin-25(0)

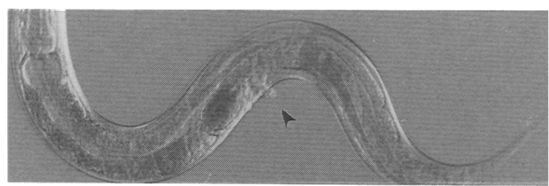

$\operatorname{lin}-I(e 1777) ; \operatorname{lin}-25(n 545 t s)$

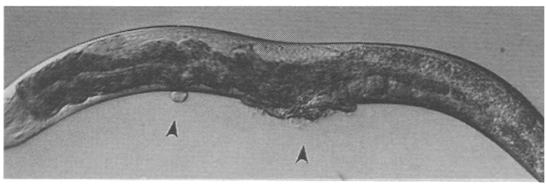

lin-I(sy254); lin-25(0) tions in lin-25. The lin-1(e1777); lin 25(n545ts) double mutant animal was raised at $20^{\circ} \mathrm{C}$, a temperature at which lin-25(n545ts) behaves as a hypomorph. lin-1(sy254) is a null allele (G. Beitel and H.R. Horvitz, pers. comm.). The lin-1(sy254); lin-25(e1446) hermaphrodite has a reduced number and size of pseudovulvae.

maphrodites in which the gonad had been ablated because in very few cases did two adjacent cells both adopt a vulval fate. However, in lin-25(0); lin-15(0) double-mutant hermaphrodites in the presence of the gonad, the cells closest to the anchor cell are often induced to adopt vulval fates. In these animals, described in more detail below, we found that lateral signaling was indeed compromised. However, in view of the fact that lin-25 is clearly required for vulval induction we favor the explanation that the inefficiency of lateral signaling seen in these animals is probably an indirect result of defects in induction.

lin-25 is required for vulval induction by activated let-60 Ras

Many hypomorphic alleles of genes encoding compo- nents of the inductive signaling pathway (including let23, sem-5, let-60, lin-45, and mpk-1/sur-1) suppress the Multivulva phenotype of lin-15(n309) (Sternberg and Horvitz 1989; Beitel et al. 1990; Han et al. 1990, 1993; Clark et al. 1992). The fact that lin-25 mutations also suppress the phenotype caused by this allele, therefore, suggested that lin-25 might be part of the same pathway. To test this idea we constructed a number of doublemutant hermaphrodites containing an activated allele of let -60 Ras, $n 1046$, and various alleles of $l i n-25$. Activated let-60 Ras alleles such as let-60(n1046) cause a Multivulva phenotype similar to that caused by lin-15(n309) (Ferguson and Horvitz 1985; Beitel et al. 1990; Han et al. 1990). We found that the lin-25 mutations ar90, n1063, e1446, n545ts, and n1722ts all suppressed the Multivulva phenotype caused by let-60(n1046) (Fig. 2). Consistent with this observation is the fact that three 
Table 3. lin-25 mutations reduce vulval induction

\begin{tabular}{|c|c|c|c|c|c|c|c|c|}
\hline Genotype & $\mathrm{AC}$ & P3.p & P4.p & P5.p & P6.p & P7.p & P8.p & Pn.p \\
\hline $\operatorname{lin}-15(n 309)^{\mathrm{a}}$ & - & $2^{\circ}$ & $2^{\circ} / 1^{\circ}$ & $2^{\circ}$ & $1^{\circ}$ & $2^{\circ}$ & $2^{\circ} / 1^{\circ}$ & \\
\hline \multirow[t]{5}{*}{$\operatorname{lin}-25(e 1446) ; \operatorname{lin}-15(n 309)$} & - & $3^{\circ}$ & $3 \%$ ind & $3 \%$ ind & $3 \%$ ind & $3 \%$ ind & $3 \%$ ind & $3^{\circ}$ \\
\hline & - & SS & SS & LOTT & S OT & SS & SLLO & SS \\
\hline & - & SLTS & SSSS & LOTO & S SS & S LL & SSSL & \\
\hline & - & SS & S & sS & LTTO & NTLL & SS & \\
\hline & - & SS & S LS & $S$ & SS & LLSS & S TS & SS \\
\hline \multirow[t]{6}{*}{ let-60(n1046) } & - & $3^{\circ}$ & $3 \%$ ind & $2^{\circ}$ & $3 \%$ ind & $2^{\circ}$ & $3^{\circ}$ & \\
\hline & - & SS & SS & $\underline{\text { LLTN }}$ & S TO & NOLL & SS & \\
\hline & - & SS & LLOO & $\underline{L L} L ?$ & OTTO & NLLL & SS & \\
\hline & - & SS & $\overline{\text { SS }}$ & $\overline{\underline{L L}} L N$ & SOT & $\underline{L L O N}$ & SS & \\
\hline & - & S LO & S OT & $\overline{\overline{L L}} L N$ & TTOT & $\overrightarrow{\text { NTLL }}$ & S SS & \\
\hline & - & SS & SS & $\overline{\mathrm{LLOT}}$ & S OO & $\mathrm{NO} \overline{\mathrm{OL}}$ & SS & \\
\hline \multirow[t]{6}{*}{ let-60(n1046); lin-25(e1446) } & - & $3^{\circ}$ & $3^{\circ}$ & $3^{\circ}$ & $3^{\circ}$ & $3^{\circ}=$ & $3^{\circ}$ & $3^{\circ}$ \\
\hline & - & SS & SS & SS & SS & SS & SS & \\
\hline & - & SS & SS & SS & SS & SS & SS & \\
\hline & - & SS & SS & SS & SS & SS & SS & SS \\
\hline & - & SS & SS & SS & SS & SS & SS & SS \\
\hline & - & SS & SS & OTTT & SS & SS & SS & SS \\
\hline
\end{tabular}

See Fig. 1 legend and Table 2 footnote for meaning of $S, N, T, L$, and $O$.

(?) Division not observed.

${ }^{\text {a}}$ From Ferguson et al. (1987).

lin-25 alleles, $k u 70, k u 77$ and $k u 78$, were isolated in genetic screens for suppressors of the let-60(n1046) phenotype ( $M$. Han, pers. comm.).

Because the expressivity of the Multivulva phenotype of let-60(n1046) is partially dependent on the anchor cell signal (P. Sternberg, pers. comm.), to determine the order in which lin-25 and let-60 function in the pathway for vulval induction, we compared the phenotype of the let60(n1046); lin-25(e1446) double mutant, with that of the single mutant, let-60(n1046), in the absence of the gonad. Twenty two of twenty three hermaphrodites of the genotype let-60(n1046) in which the gonad had been ablated were Multivulva. In contrast, all 35 animals of the genotype let-60(n1046); lin-25(e1446) in which the gonad had been ablated were non-Multivulva. The suppression of the Multivulva phenotype of let-60(n1046) was reflected in the fates adopted by the VPCs. In let60(n1046) animals in which the gonad had been ablated, many VPCs still adopted vulval fates (Table 3), whereas in let-60(n1046); lin-25(e1446) double-mutant animals in which the gonad had been ablated almost all VPCs adopted the nonvulval fate (Table 3 ). These results suggest that lin-25 functions after let-60 in the genetic pathway for vulval induction.

lin-25 may be required for the induction of a component of the lateral signaling pathway

lin-25 mutations do not completely abolish the ability of the VPCs to respond to the anchor cell signal. In the lin-25 single mutant, and in double-mutant hermaphrodites of the genotypes let-60(n1046); lin-25(e1446), and lin-25(e1446); lin-15(n309) in which the gonad is intact, cells close to the anchor cell are often induced to adopt vulval cell fates (Table 4). In the double-mutant hermaphrodites, we observed a number of instances in which two adjacent VPCs both adopted the $1^{\circ}$ fate. Of 19 pairs of adjacent cells (in 13 different animals) in which both cells adopted a vulval fate we observed 5 pairs in which both members of the pair adopted the $1^{\circ}$ fate $(\mathrm{Ta}$ ble 4). In hermaphrodites of the genotype lin-15(n309) the frequency with which adjacent VPCs both adopt the $1^{\circ}$ fate is considerably lower (1/69 pairs of cells) (Sternberg 1988; Sternberg and Horvitz 1989). Therefore, lin-25 mutations affect the efficiency with which VPCs signal to one another laterally. One possible explanation for this observation is that lin-25 is required for the efficient expression of some component of the lateral signaling pathway in response to Lin-3. Alternatively, lin-25 could be required at two separate points during VPC fate specification, first as a component of the vulval induction pathway and second as a component of the lateral signaling pathway.

\section{Genetic interactions between lin-25 and genes acting after mpk-1/sur-1 MAPK in the pathway for vulval induction}

All existing alleles of $l i n-25$ reduce the efficiency of vulval induction. Because this is also true for both mpk-1/ sur-1 MAPK and lin-45 Raf it was not possible to perform epistasis tests between lin-25 and either of these genes. Multivulva mutations exist, however, in two genes, lin-1 and lin-31, that function after $m p k-1 /$ sur-1 in the genetic pathway for VPC fate specification.

lin-1 encodes a negative regulator of vulval induction: Loss-of-function alleles of lin-1 display a Multivulva phenotype similar to that caused by lin-15(n309) (Hor- 
Table 4. lin-25 may be required for the induction of a component of the lateral signaling pathway

\begin{tabular}{|c|c|c|c|c|c|c|c|c|}
\hline Genotype & $\mathrm{AC}$ & P3.p & P4.p & P5.p & P6.p & P7.p & P8.p & Pn.p \\
\hline let $-60(n 1046)^{a}$ & + & $1 \% / 2^{\circ} / 3^{\circ}$ & $1^{\circ} / 2^{\circ}$ & $2^{\circ}$ & $1^{\circ}$ & $2^{\circ}$ & $1 \% / 2^{\circ} / 3^{\circ}$ & \\
\hline let-60(n1046); & + & SS & SS & SS & \begin{tabular}{|l|} 
TLTT \\
\end{tabular} & \begin{tabular}{|l|} 
TTTT \\
\end{tabular} & SS & SS \\
\hline \multirow[t]{7}{*}{$\operatorname{lin}-25(e 1446)$} & + & SS & SS & $\underline{\text { LLTN }}$ & TTTO & NTLL & SS & \\
\hline & + & SS & SS & S OT & TTTT & NOLL & SS & \\
\hline & + & SS & SS & S TT & OOTO & ss - & SS & SS \\
\hline & + & S & SS & \begin{tabular}{|l|} 
TOTT \\
\end{tabular} & \begin{tabular}{|l|} 
TTOL \\
\end{tabular} & OLLL & SS & SS \\
\hline & + & SS & SS & S TT & OOTO & SS & SS & SS \\
\hline & + & SS & SS & LNTO & TTTT & NTLL & SS & SS \\
\hline & + & SS & S & SS & $\mathrm{L} ? \mathrm{TT}$ & TT S & SS & $\mathrm{S}$ \\
\hline $\operatorname{lin}-15(n 309)$ & + & $2^{\circ}$ & $2^{\circ} / 1^{\circ}$ & $2^{\circ}$ & $1^{\circ}$ & $2^{\circ}$ & $1 \% 2^{\circ}$ & \\
\hline $\operatorname{lin}-25(e 1446)$ & + & $S$ & SS & OTLO & \begin{tabular}{|l|} 
TTTT \\
\end{tabular} & OTTT & SS & \\
\hline \multirow[t]{4}{*}{$\operatorname{lin}-15(n 309)$} & + & SS & SS & $\underline{\text { LLTN }}$ & TTTT & NTLL & $\mathrm{S}$ & \\
\hline & + & $\mathrm{S}$ & SS & S TO & LOTT & TTTT & NTLLL & SS \\
\hline & + & S & SS & LLTT & \begin{tabular}{|l|l|} 
LTTT \\
\end{tabular} & \begin{tabular}{|l|} 
TTTL \\
\end{tabular} & NOLL & SS \\
\hline & + & S & SS & OTTT & TOTT & TTLL & SSSS & SS \\
\hline
\end{tabular}

See Fig. 1 legend and Table 2 footnote for the meaning of S, N, T, L, and O.

(?) Division not observed.

${ }^{a}$ From Ferguson et al. (1987). For animals of the genotypes lin-25(e1446); lin-15(n309) and let-60(n1046); lin-25(e1446), boxes highlight cases of adjacent $1^{\circ}$ lineages. Not all $1^{\circ}$ lineages are boxed.

vitz and Sulston 1980; Ferguson et al. 1985). Reduction of lin-1 activity causes a Multivulva phenotype even if let-60 Ras, lin-45 Raf, or mpk-1/sur-1 MAPK activity is reduced (Han et al. 1990, 1993; Lackner et al. 1994; Wu and Han 1994; S. Tuck and I. Greenwald, unpubl.). We constructed many different lin-1; lin-25 double mutants with alleles of varying severity of each gene. We found that all lin-25 alleles tested, including the weakest allele, lin-25(n1722), suppressed the Multivulva phenotype caused by lin-1(e1275) and lin-1 (e1777am) (Horvitz and Sulston 1980; Ferguson and Horvitz 1985). lin$1(e 1777 \mathrm{am})$ strongly reduces lin-1 activity (Horvitz and Sulston 1980; Ferguson and Horvitz 1985). However, lin25(e1446) only weakly suppresses the Multivulva phenotype of the lin-1 molecular null alleles lin-1(ar147) and lin-1(sy254) (kindly provided by G. Beitel and H.R. Horvitz, MIT, Boston, MA). Double mutants of genotype lin-1(sy254); lin-25(e1446) display a slight reduction in the size and number of pseudovulvae (Fig. 2), although lineage analysis indicates that the double mutant has considerably more induction than does lin-1(e1777); lin25 (data not shown).

lin-31 mutations cause a complex phenotype in which aspects of the Multivulva and Vulvaless phenotypes may both be expressed within the same animal. One or more of the VPCs that normally adopt a nonvulval fate, P3.p, P4.p, and P8.p, instead adopt a vulval fate, and one or more of the VPCs that normally adopt a vulval fate, P5.p, P6.p, and P7.p, instead adopt the nonvulval fate. It is not yet known where in the pathway for VPC fate specification lin-31 acts in relation to lin-1. Most $(77 \%)$ lin31(n301) hermaphrodites are Multivulva (Miller et al. 1993). We found that in lin-31(n301); lin-25(e1446) double-mutant hermaphrodites, the size and number of pro- trusions were slightly reduced but that the majority of animals $(73 \%, n=186)$ were still Multivulva.

\section{lin-25 encodes a novel protein}

To begin to explore the biochemical function of lin-25 we cloned and sequenced the gene. lin-25 mapped to an $\sim 2.5 \mathrm{Mb}$ interval between myo-3 and TcPAR2, a restriction fragment length polymorphism (RFLP) (Fig. 3A). We further correlated the physical and genetic maps in the region by identifying, mapping, and cloning a number of Tcl-associated RFLPs. Two of these, arP2 and arP5, lying respectively to the right and left of $l i n-25$ (Fig. $3 \mathrm{~A}$ and B), defined an interval of $\sim 250 \mathrm{~kb}$. Cosmid and $\lambda$ phage clones (Coulson et al. 1986, 1988, pers. comm.) in this interval were pooled and injected into the germ line of lin-25 mutant hermaphrodites and tested for their ability to complement the egg-laying defect (Fig. 3C). Ultimately, a $10.1-\mathrm{kb}$ Spel fragment from the $\lambda$ phage clone YSL59 was found that complemented efficiently. Deletion of $\sim 2 \mathrm{~kb}$ from either end of this fragment abolished the rescuing activity (Fig. $3 \mathrm{C}$ ).

When the $10.1-\mathrm{kb}$ fragment was used to probe Northern blots containing RNA from a mixed stage population of $C$. elegans hermaphrodites, two poly $(\mathrm{A})^{+}$transcripts were detected: One of $\sim 3.6 \mathrm{~kb}$ (Fig. 3D) and a second of $\sim 800 \mathrm{bp}$ (not shown). No transcripts were detected in the poly $(\mathrm{A})$ - fraction. The $3.6 \mathrm{~kb}$ transcript appeared likely to correspond to the lin-25 gene because probes throughout the $10.1-\mathrm{kb}$ rescuing fragment hybridized to the 3.6$\mathrm{kb}$ transcript, whereas only those containing an 850-bp fragment from one end $\left(3^{\prime}\right.$ of the $3.6-\mathrm{kb}$ transcript $)$ hybridized to the 800-bp transcript (not shown). The 10.1$\mathrm{kb}$ fragment was completely sequenced on both strands. 

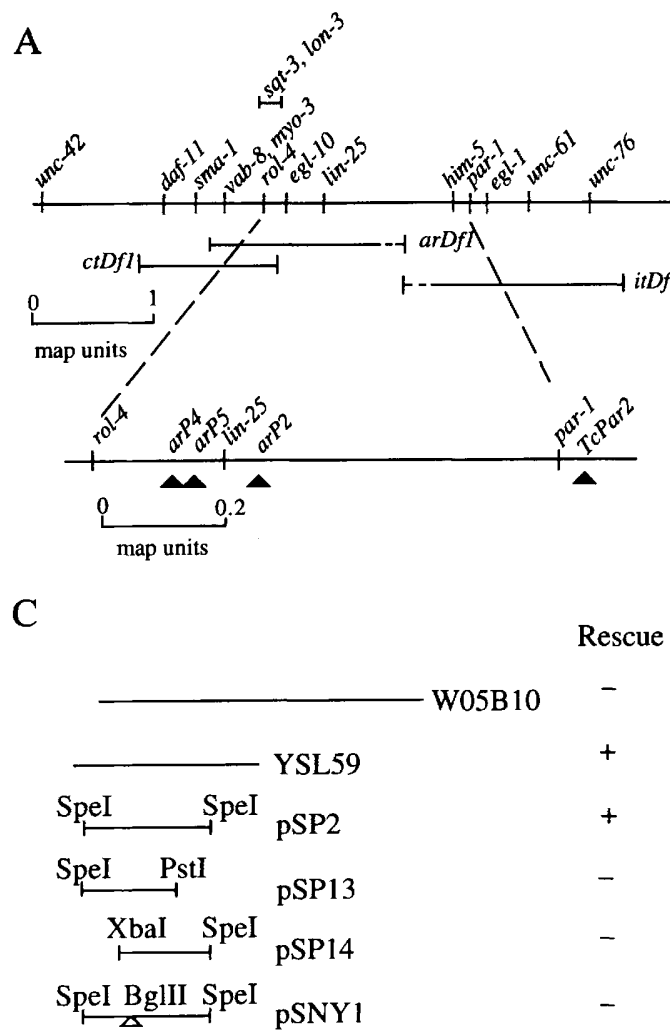

B

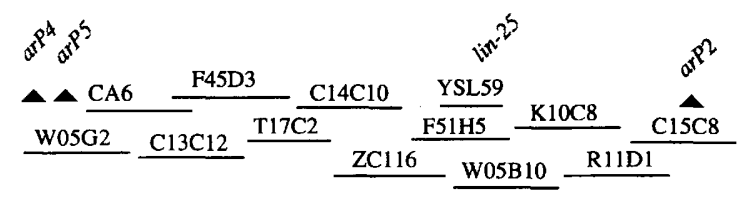

$\mathrm{D}$

Figure 3. Genetic and physical maps of regions around lin-25. (A) Partial genetic map of the lin-25 $\mathrm{V}$ region. The duplication $c t D p 11$, not shown, complements unc-42, unc-76, and all the markers lying between (Hunter and Wood 1992). arP2, arP4, and arP5 are Bristol $(\mathrm{N} 2) /$ Bergerac $(\mathrm{BO})$ Tc1-associated RFLPs. $(B)$ Physical map of the region around lin-25. YSL59 is a $\lambda$ phage clone. All others are cosmid clones. Not all clones on the physical map are shown. (C) Plasmid, cosmid, and $\lambda$ phage clones in the immediate vicinity of lin-25. Their ability to rescue the temperature sensitive mutation, lin-25(n545ts) is indicated on the right. W05B10 and YSL59 are cosmid and $\lambda$ phage clones, respectively; the rest are plasmid clones containing inserts derived from YSL59. pSNY1 contains a linker insertion at a unique BglII site in exon 7. (D) The structure of the lin-25 gene. Large boxes represent exons. Solid regions represent the putative protein coding region starting at an ATG in exon 1 and ending at TGA in exon 13. Products resulting from the splicing of the SLl spliced leader RNA to the $5^{\prime}$ ends of exons 1,2 , and 3 have been observed as have those resulting from the splicing of SL2 to the same exons. Smaller boxes below represent cDNA clones. Shaded areas represent regions of each clone that were sequenced. All SpeI, PstI, $B g l l I$, and $X b a I$ sites are shown.

Several cDNAs corresponding to the $3.6-\mathrm{kb}$ transcript were also isolated and sequenced (see Materials and methods). We confirmed that the $3.6-\mathrm{kb}$ transcript corresponds to the lin-25 gene in two ways. First, we introduced a linker-insertion mutation into a unique BglII site in an exon on the 10.1-kb rescuing fragment (Fig. $3 \mathrm{D} \mid$. The linker contained a stop codon in all three frames and completely abolished the rescuing activity (Fig. 3C). Second, we have identified molecular lesions in two lin-25 alleles, ar90 (codon 197: CAG $\rightarrow$ TAG) and ga67 (codon 284: TTC $\rightarrow$ TAC).

A conceptual cDNA 3659 nucleotides in length appears to span the entire gene. A putative translation initiation codon at position 55 lies in a favorable context (Kozak 1986) and is preceded by a stop codon, in frame, at position 49 . At the $3^{\prime}$ end, there are $15 \mathrm{dA}$ residues, not present in the genomic sequence, located 16 nucleotides from a potential polyadenylation signal sequence, TATAAA (Birnstiel et al. 1985). The 5' end contains spliced leader sequences (see Materials and methods).
Comparison of the cDNA and genomic sequences revealed that all 13 exons are present on the $10.1-\mathrm{kb}$ rescuing fragment (Fig. 3D). The size of the composite cDNA is consistent with the size of the mRNA estimated by Northern blot analysis (Fig. 4).

Conceptual translation of the open reading frame generated a polypeptide sequence of 1139 amino acids. Both the nucleotide sequence and deduced amino acid sequence are shown in Figure 5. The protein sequence was compared to that of all proteins in the GenBank and EMBL data bases using both the BLAST (Altschul et al. 1990 ) and FASTA (Pearson and Lipman 1988) programs. No significant similarity to other proteins was detected. With the exception of potential sites for posttranslational modification, no matches to known peptide sequence motifs were detected by use of the computer programs from the GCG (Devereux et al. 1984) and MacVector (IBI) software packages.

Lin- 25 contains a single match to the full MAPK phosphorylation site consensus, PX/S/T)P (Clark-Lewis et al. 


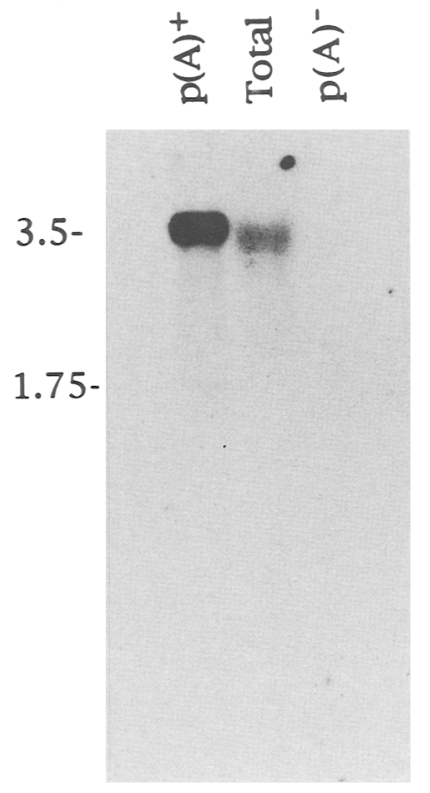

Figure 4. Northern blot analysis of the lin-25 transcripts. RNA was isolated from a mixed developmental stage population of wild-type $C$. elegans hermaphrodites. The positions to which the $28 \mathrm{~S}(3.5 \mathrm{~kb})$ and $18 \mathrm{~S}(1.75 \mathrm{~kb})$ rRNAs migrated is indicated at left. The blot was probed under high stringency conditions with radiolabeled RNA made in vitro with lin-25 cDNAs as templates for transcription. lin-25 sequences represented in the probe extend from position 245 (Fig. 5 ) to position 3565 .

1991; Gonzalez et al. 1991|, and threc additional matches to the core consensus, $[\mathrm{S} / \mathrm{T}) \mathrm{P}$. A single phosphorylation site is sufficient for the regulation of some proteins by MAPK (O'Neill et al. 1994; Brunner et al. 1994b/ but the probability that the consensus occurs purely by chance in a protein the size of Lin-25 is quite high $\quad \sim 0.3$ for the full consensus, assuming a random distribution of amino acids occurring at the frequency they are found in Lin-25). Lin-25 also contains a potential nuclear localization signal (Boulikas 1993) (Fig. 5).

As mentioned above, the lin-25(ar90) allele, which is suppressed by the amber suppressor tRNA sup-7(st5) (Waterston 1981; Wills et al. 1983) is associated with an amber mutation at codon 197 . This mutation would lead to the production of a severely truncated protein $<20 \%$ the size of the predicted 1139-amino-acid Lin-25 protein. This observation strongly supports the genetic arguments that lin-25(ar90) is a null allele.

\section{Discussion}

Genetic analysis of vulval development in the C. elegans hermaphrodite has shown that the induction of vulval fates is mediated by a signal transduction pathway involving let-60 Ras, lin-45 Raf, and the mpk-1/sur-1 MAPK (Han and Sternberg 1990; Han et al. 1993; Lackner et al. 1994; Wu and Han 1994). In this study, we have shown that the absence of lin-25 activity causes a de- crease in the amount of vulval induction. The genetic interactions with other mutations affecting vulval induction suggest that lin-25 acts after the Raf/MAPK protein kinase cascade and that a decrease in vulval induction may lead to a decrease lateral signaling, a lin-12mediated signaling event.

lin-25 appears to function after let-60 Ras in the genetic pathway for vulval induction

Dominant mutations in let-60 Ras, resulting from changes in codon 13 , constitutively activate the biochemical pathway that mediates the response to the vulva inducing signal Lin-3 (Beitel et al. 1990; Han et al. 1990; Han and Sternberg 1990). Hermaphrodites carrying such mutations display a Multivulva phenotype resulting from fate transformations in which cells in the ventral hypodermis that normally adopt the hypodermal fate instead adopt vulval fates. Like partial loss-of-function mutations in lin-45 Raf (Han et al. 1993) and the mpk1/sur-1 MAPK (Miller et al. 1993; Wu and Han 1994), mutations in lin-25 that reduce or eliminate the activity of the protein suppress the Multivulva phenotype of let60(n1046) activated Ras.

It seems unlikely that $l i n-25$ is an immediate target of Let-60 Ras during vulval induction. Biochemical experiments on cultured cells and genetic experiments in Drosophila have shown that two immediate targets of Ras in both mammals and flies are the proteins Raf and MEK (Tsuda et al. 1993; Van Aclst et al. 1993; Vojtek et al. 1993; Warne et al. 1993; Zhang et al. 1993). Furthermore, evidence from many different studies suggests that the pathway between Ras and MAPKs is a simple lincar one (Ambrosio et al. 1989; Crews et al. 1992; Kyriakis et al. 1992; Thomas et al. 1992; Wood et al. 1992; Lu et al. 1993; Ogiso et al. 1993; Tsuda et al. 1993; Brunner et al. 1994a; Leevers et al. 1994; Stokoe et al. 1994). Both Raf and a MAPK are involved in vulval induction (Han et al. 1993; Lackner et al. 1994; Wu and Han 1994), suggesting that components of this highly conserved biochemical pathway perform similar functions. It therefore seems likely that lin-25 functions further downstream in the pathway, after mpk-1/sur-1 MAPK. Although we could not perform epistasis experiments to test this idea directly, because all existing mutations in lin-45 Raf, mpk-1/sur-1 MAPK, and lin-25 reduce vulval induction, this interpretation is supported by the genetic interactions between lin-25 and lin-1, a gene that is thought to act after mpk-1/sur-1 (Lackner et al. 1994; Wu and Han 1994). While lin-1 is epistatic to mutations in let-60 Ras, lin-45 Raf, and mpk-1/sur-1 MAPK, lin-25 mutations suppress the multivulva phenotype caused by certain alleles of lin-1. The simplest interpretation of our results therefore is that lin-25 acts downstream of the mpk-1/ sur-1 MAPK, after or at the same time as lin-1.

One explanation for the observation that lin-25 is epistatic to let-60(n1046) would be that lin-25 is necessary for the VPCs to be intrinsically capable of adopting vulval fates. We think it unlikely, however, that lin-25 is involved solely in establishing the identity of the VPCs 


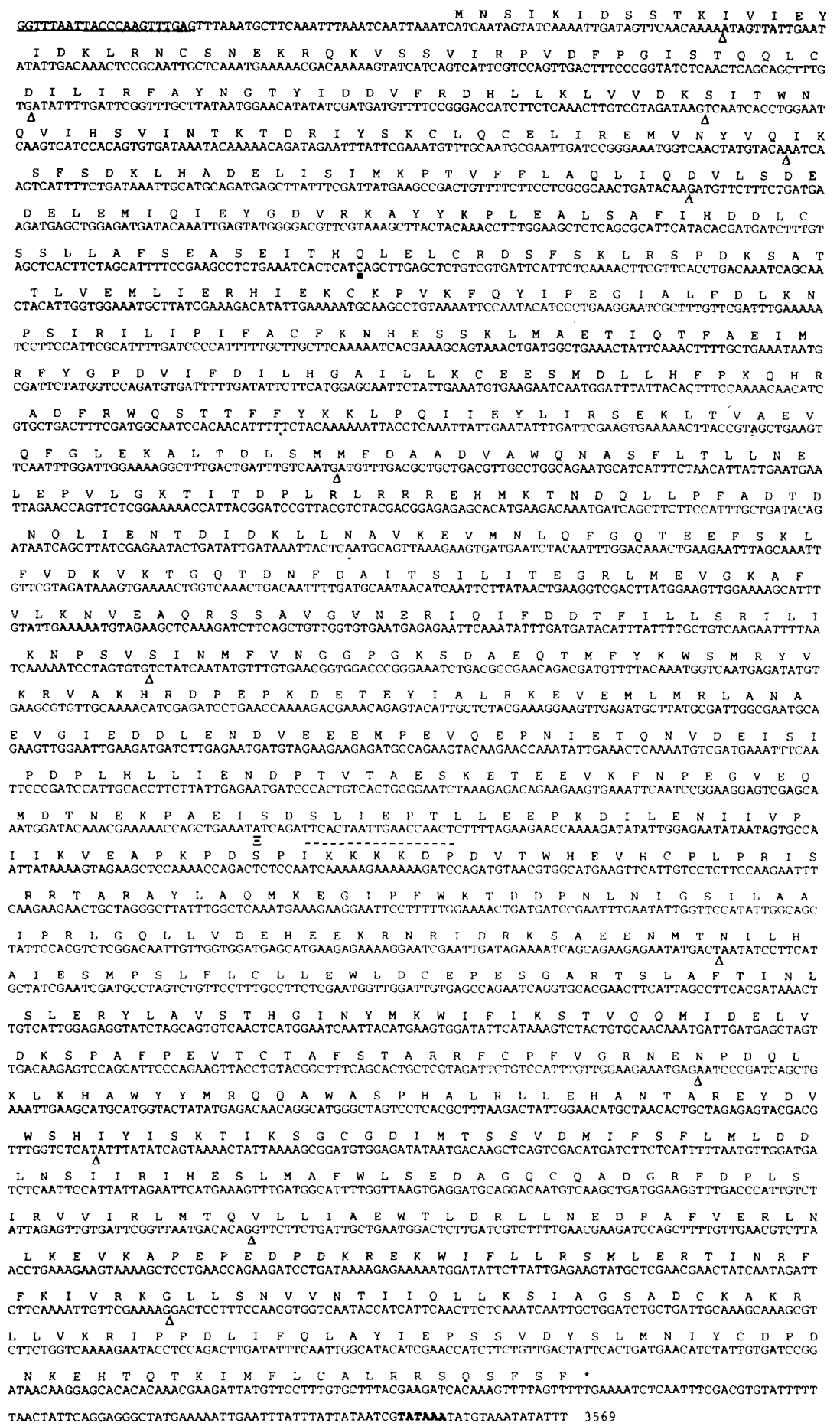

${ }_{100}^{16}$

200

${ }_{300}^{82}$ ${ }_{400}^{116}$ ${ }_{509}^{149}$ $(500$ 182
600 ${ }_{700}^{216}$ ${ }_{800}^{249}$ 282 316
1000 ${ }_{1100}^{349}$ 382 1200 ${ }_{1300}^{416}$ 449 482 482
1500 516
1600 549
1700 582
1800 616 1900 649
2000 682 2100 716
2200 749 782
2400

Figure 5. lin-25 cDNA sequence and deduced amino acid sequence. Amino acid numbers are shown at the top and nucleotide numbers at the bottom. The termination codon TGA is denoted by an asterisk. The SLl trans-spliced leader sequence is underlined. A potential polyadenylation signal sequence is shown in bold. $(\Delta)$ Intron-exon boundaries. A potential MAPK phosphorylation site, PX(S/T)P |ClarkLewis et al. 1991; Gonzalez et al. 1991), is denoted by $\Xi$, and a potential nuclear localization signal sequence (Boulikas 1993) is overlined with dashes. Codon 197, which is altered by lin-25(ar 90$)$ to an amber codon, is indicated by a solid square positioned underneath. because P6.p often adopts the $1^{\circ}$ fate in lin-25 mutants (Ferguson et al. 1987; this work) and VPCs adopt the $2^{\circ}$ fate in lin-12(d); lin-25 double mutants. These results imply that lin-25 mutations do not affect the identity of the VPCs in such a way that they are unable to adopt vulval fates per se.

The position of lin-25 downstream of let-60 Ras in the genetic pathway suggests a number of possible functions for the novel predicted Lin-25 protein. Because many of the changes induced by the Ras/MAPK pathway (in all cells) undoubtedly result from changes in gene expression it is possible that Lin-25 is a transcription factor required for the induction of genes in response to Lin-3. Another possibility is that Lin-25 does not itself bind DNA but modulates the activity of a protein which does; for example, we predict that there will be a C.elegans protein similar to MCMl and SRF (Treisman and Ammerer 1992). Finally, it is possible that Lin-25 and other 
proteins acting after Let- 60 in the vulval induction pathway may act to cause changes in cell physiology directly rather than through changes in gene expression.

It is possible that another gene is partially functionally redundant with lin-25. Alleles that strongly reduce or eliminate lin-25 activity lead to high-penetrance reduction in vulval induction (and an apparently unrelated defect in egg-laying; see Materials and methods) but only low-penetrance sterility, larval lethality, and extra VPCs formation from P3.p-P8.p doublings (see Table 1 and Materials and methods). In contrast, alleles that strongly reduce or eliminate let-60 Ras, lin-45 Raf, or mpk-1/ sur-1 MAPK lead to sterility or a highly penetrant larval arrest with a characteristic rod-like morphology (Ferguson et al. 1985; Beitel et al. 1990; Han et al. 1990, 1993; Lackner et al. 1994; Wu and Han 1994). These observations may indicate that another gene is functionally redundant with lin-25 for the sterility and larval lethal defects. There may also be functional redundancy for vulval induction because P6.p is generally induced in lin-25 mutants.

\section{lin-25, lin-1, and lin-31 may not function in a} simple linear pathway

Besides lin-25 and lin-1, one other gene, lin-31, has been shown to act after $m p k-1 /$ sur-1 in the genetic pathway for VPC fate specification. It is not likely, however, that lin-1, lin-25, and lin-31 function in a simple linear biochemical pathway. The phenotypes caused by mutations in $l i n-1$, lin-25, and $l i n-31$ and the interactions observed among these mutations suggest that the Lin-1, Lin-25, and Lin-31 proteins may not directly regulate one another. Hermaphrodites carrying null mutations in lin-31 show aspects of both the Vulvaless and Multivulva phenotypes (Ferguson et al. 1987; Miller et al. 1993). P5.p, P6.p, or P7.p sometimes adopts the nonvulval fate and P3.p, P4.p, or P8.p sometimes adopts a vulval fate. This phenotype implies that Lin-25 cannot function solely by regulating the activity of Lin-31 for vice versa) because lin-25, unlike $\operatorname{lin}-31$, is not required for the specification of the $3^{\circ}$ fate. Similarly it is not possible that Lin-1 acts solely by regulating the activity of Lin-31 (or vice versa) because lin-1, unlike lin-31, does not appear to be required for the specification of the vulval fates. A further reason to think that $\operatorname{lin}-1, \operatorname{lin}-25$, and $\operatorname{lin}-31$ might not directly regulate one another is the fact that there is evidence to suggest that more than one target may exist for the mpk-1/sur-1 MAPK (G. Beitel, S. Tuck, I. Greenwald and H.R. Horvitz, in prep.).

While lin-1, lin-31, and lin-25 may not act in a linear pathway, it is possible that they act together in certain aspects of VPC fate specification. lin-31 encodes a member of the HNF3/forkhead family of transcription factors (Miller et al. 1993). To account for the lin-31 mutant phenotype Miller and co-workers have proposed that transcription complexes exist in the VPCs that can activate vulval specific genes and repress hypodermal ones and that others exist that can repress vulval specific genes and activate hypodermal ones (Miller et al. 1993).
The deregulation of cell fate specification seen in animals carrying lin-31 mutations could be explained if Lin31 were part of both types of complex. There would be inefficient repression of vulval specific genes in P3.p, P4.p, and P8.p but at the same time inefficient activation of such genes in P5.p, P6.p, and P7.p. This model requires that Lin-31 acts in concert with other proteins to specify cell fates. It is possible that Lin-25 and Lin-1 each function together with Lin-31 to control the expression of the vulval and nonvulval fates, respectively.

Genetic epistasis experiments between lin-25 and lin-1 suggest a complex, dosage-sensitive relationship between the two genes. All alleles of lin-25, including lin-25(n1722), which itself has little effect on vulval induction, suppress the Multivulva phenotypes caused by lin-1(e1275) and lin-1(e1777), which strongly reduce lin-1 activity (Ferguson et al. 1985). However, lin25(e1446) has only mild effects on the ectopic vulval induction caused by lin-1 molecular null alleles $/ \mathrm{G}$. Beitel and H.R. Horvitz, pers. comm.; data not shown). One possible explanation for these interactions would be that downstream of mpk-1/sur-1 MAPK lin-1 and lin-25 do not regulate one another directly but instead act on separate branches of a forked pathway. MAPKs are capable of phosphorylating a number of different proteins, and it has been suggested that MAPK may act at a point at which signal transduction pathways frequently branch (Stokoe et al. 1992). Vulval induction, therefore, might be the result of two different activities of $s u r-1 / \mathrm{mpk}-1$ MAPK: inactivation of Lin-1 and activation of Lin-25. This model and a number of others also consistent with the experimental data are presented in Figure 6.

The interactions between $l i n-1$ and $l i n-25$ are reminiscent of the interactions between yan and pointed (ptd), two genes involved in R7 photoreceptor cell fate determination in Drosophila (Lai and Rubin 1992; Brunner et al. 1994b). O'Neill et al. (1994) and Brunner et al. (1994b) have suggested that the combination of positive modulation of $\mathrm{PtdP} 2$ and negative modulation of Yan provides more delicate control of induction than could be achieved if just one target existed. The combination of the negative factor Lin-1 and the positive factor Lin-25 may similarly provide sensitive control over vulval induction in C. elegans.

\section{lin-25 may help link two different signaling pathways in the VPCs}

In the wild-type hermaphrodite the vulva is formed from the descendants of the three VPCs closest to the anchor cell, P5.p, P6.p, and P7.p. P6.p adopts one vulval fate, the $1^{\circ}$ fate, whereas P5.p and P7.p adopt a different vulval fate, the $2^{\circ}$ fate (Sulston and Horvitz 1977). The specification of the $2^{\circ}$ fate is controlled by lateral signaling, an event thought to occur between adjacent VPCs (Sternberg 1988; Sternberg and Horvitz 1989|. Lateral signaling, which is mediated by the receptor lin-12, ensures that adjacent VPCs do not both adopt the $1^{\circ}$ fate (Greenwald et al. 1983; Sternberg and Horvitz 1989).

The phenotypes of certain double mutants suggests 
A

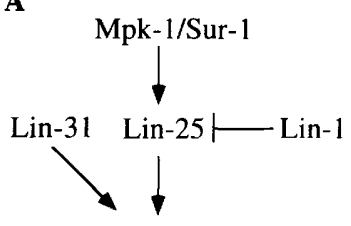

vulval fates

C

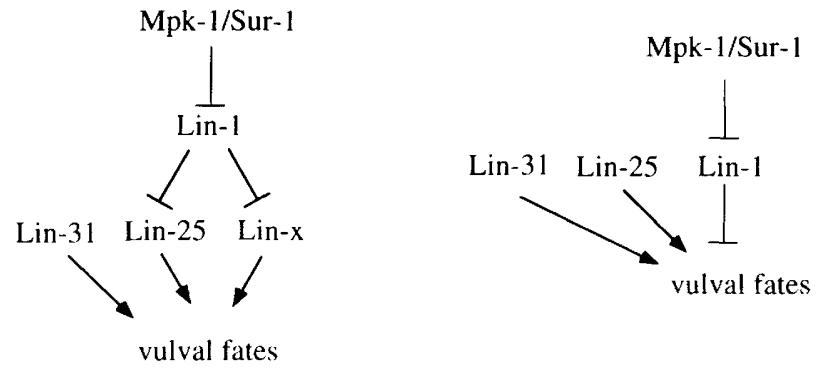

Figure 6. Models for how Lin-25 might function during VPC fate specification. In models $A, B$, and $C$, Lin- 25 could either be a direct target of Mpk-1/Sur-1/or some intervening kinase) or be required for the activity of the direct target. The phenotypes of mutations in lin-1, lin-25, and lin-31 imply that Lin-25 is formally a positive regulator of vulval fates (Ferguson et al. 1987), Lin-1 is a negative regulator (Horvitz and Sulston 1980; Sulston and Horvitz 1981; Ferguson and Horvitz 1985) and Lin-31 is required for both vulval and nonvulval fates (Ferguson et al. 1987; Miller et al. 1993). (A) Lin-25 is a substrate for the Mpk1/Sur-1 MAPK (or some intervening kinase). Lin-1 negatively regulates Lin- 25 activity. Lin-31 acts in conjunction with Lin- 25 to specify vulval fates and with some other protein to specify nonvulval fates (not shown). (B) Both Lin-1 and Lin-25 are targets of Mpk-1/Sur-1 (or some intervening kinase). This model is similar to the one proposed for the function of Yan and PtdP2 in the determination of the R7 photoreceptor cell fate in Drosophila (O'Neill et al. 1994; Brunner et al. 1994b). (C) Lin-1, a target of the pathway, negatively regulates Lin- 25 activity. Because lin-25 mutations only weakly suppress null mutations in lin-1 (data not shown), for this model to be correct, lin-1 must also regulate another gene, here denoted $l i n-x$, with which lin25 is partially redundant. No genes with this characteristic have yet been identified. $(D)$ Lin- 25 acts in a pathway parallel to Lin-1 and $\operatorname{Lin}-31$.

that lin-25 may help link the inductive and lateral signaling pathways. lin-25 mutations do not completely abolish the ability of the VPCs to respond to the inducing signal, Lin-3: In lin-25 mutant hermaphrodites P5.p and P7.p adopt the nonvulval $\left(3^{\circ}\right)$ fate but P6.p often adopts the $1^{\circ}$ fate (Ferguson et al. 1987). In double-mutant hermaphrodites containing both a lin-25 mutation and a mutation that constitutively activates the inductive signaling pathway two or three VPCs closest to the anchor cell are often induced to adopt vulval fates. In these animals the efficiency of lateral signaling appears to be reduced because two adjacent VPCs sometimes both adopt the $1^{\circ}$ fate. In view of the fact that lin-25 mutations affect vulval induction, the most likely explanation for this observation is that $l i n-25$ is required for the expression or activation of some component of the lateral signaling pathway. Dominant mutations in lin-12 are epistatic to mutations in lin-25. Therefore, lin-25 mutations may reduce the efficiency of lateral signaling by affecting the synthesis of the ligand for Lin-12. In this model, the lin-25 mutant phenotype, the failure of P5.p and P7.p to adopt the $2^{\circ}$ fate, could be the result of two separate defects, both essentially caused by a decrease in the amount of induction. First, P5.p and P7.p are less sensitive to the lateral signal from P6.p, because they have not been adequately induced by the anchor cell, and second, P6.p has a reduced ability to signal.

\section{Materials and methods}

Strains and genetic analysis

The methods used for the culturing, handling, mutagenesis, and genetic manipulation of $C$. elegans were those described by Brenner (1974). Experiments were performed at $20^{\circ} \mathrm{C}$ except as otherwise noted. C. elegans var. Bristol strain N2 is the wildtype parent for most of the strains used in this work. The only exceptions are the congenic strains DT 127 and GS352 in which part of the right arm of chromosome $\mathrm{V}$ is derived from C. elegans var. Bergerac (Nigon 1949).

We generated arDf1 during the course of this work by X-ray mutagenesis of a chromosome marked with unc-42(e270) sqt3(sc63). arDf1 fails to complement vab-8(e1017), sqt-3(sc63ts), lon-3(e2175), and lin-25(n545ts). It complements sma-1(e30), par-1(b274mat), him-5(e1467), itDf2, unc-61(e228), and unc76(e911). arDf1 complemented all visible genetic markers to the right of lin-25 that we tested. However, by Southern blotting we found that $\operatorname{arDf1}$ extends to the right of $\operatorname{arP} 2$, which itself lies $\sim 90 \mathrm{~kb}$ to the right of lin-25 (Fig. 3B,C).

To isolate Iin-25(ar 90$)$, hermaphrodites of the genotype unc42 (e270) sqt-3(sc63ts), grown at $15^{\circ} \mathrm{C}$, were exposed to ethyl methanesulfonate (EMS) (Brenner 1974) and then mated to lon$3(e 2175)$ lin-25(n545ts) males (also grown at $15^{\circ} \mathrm{C}$ ). Rol non-Unc $F_{1}$ cross progeny hermaphrodites, grown at $25^{\circ} \mathrm{C}$, were screened for fertile animals that were unable to lay eggs (the bag-ofworms phenotype|. A single new lin-25 allele, ar 90 , was isolated from a screen of $\sim 20,000$ hermaphrodites. For technical reasons, we think that this frequency is an underestimate of the actual frequency with which lin-25 alleles may be generated. lin-25(n1063) was isolated using a similar scheme from a screen of 5,000 hermaphrodites (K. Edwards and H.R. Horvitz, pers. comm.).

lin-25(e1446), lin-25(n1063), and lin-25(ar90) were tested for suppression by sup-7(st5), an amber suppressor tRNA (Waterston 1981; Wills et al. 1983). sup-7(st5) suppresses the egg-laying defect of $\operatorname{lin}-25(a r 90): 50 \%$ of hermaphrodites of the genotype lin-25(ar90); unc-6(e78)/sup-7(st5) grown at $20^{\circ} \mathrm{C}$ were able to lay eggs $(n=30)$. One hundred percent of animals of the genotype lin-25(ar90); sup-7(st5) grown at $22^{\circ} \mathrm{C}$ were able to lay eggs.

\section{lin-25 alleles and their phenotypes}

The mutations ar $90, n 1063, k u 70, k u 77, k u 78$, sy29, ga67, and $\mathrm{n} 1722$ are lin-25 alleles by the following criteria. All eight $\mathrm{mu}$ tations fail to complement lin-25(n545ts) for the egg-laying defect. ar $90, n 1063$, sy29, and $n 1722$ map to the interval between 
rol-4 and par- 1 on chromosome V. ku70, ku77, and ku 78 all map to the right of unc-42 on chromosome V. arDf1 fails to complement all eight mutations for the egg-laying defect.

Fertility and viability Hermaphrodites homozygous for strong lin-25 mutations are sometimes sterile (Ferguson and Horvitz 1985; see Table 1). The sterility defect is associated with heterogeneous gonadal and germ-line anatomy, but in some animals, the germ-line phenotype of lin-25 mutant hermaphrodites resembles that of let-23 partial loss-of-function mutants (E. Lambie, pers. comm.). We have found that strong lin-25 mutations also cause a low penetrance larval lethality (Table 1). Approximately six percent of hermaphrodites homozygous for ar90, e1446, n1063, ku70, ku77, ku78, or ga67 arrest at early larval stages (Table 1). We do not yet know the cellular basis for this phenotype. Many of the animals arrest during the Ll stage and assume a characteristic rod shape. At low magnification the arrested animals appear similar to those carrying strong mutations in genes required for vulval induction such as lin-3, let-60, and let-23 (Ferguson and Horvitz 1985; Beitel et al. 1990; Han et al. 1990). The cause of death of these animals is also not known.

Egg-laying Temperature-shift experiments indicate that lin-25 is required during the early L4 stage for the development of some component of the egg-laying apparatus in addition to its role in VPC fate determination (Ferguson et al. 1987). There is good evidence to suggest that the high penetrance of the egglaying defect caused by lin-25 mutations is mostly a reflection of this later role. In double-mutant hermaphrodites of the genotype let-60(n1046); lin-25(e1446) the VPC lineage defects caused by the two mutations are often mutually suppressed (Table 4). Interestingly, however, even when the VPC lineages are wild-type, the animals are still completely unable to lay eggs. Furthermore, although the temperature-sensitive allele, lin-25(n.545ts), is a very good suppressor of the Multivulva phenotype caused by let-60(n1046) even at the semipermissive temperature $\left(20^{\circ} \mathrm{C}\right)$, the reverse is not true: let-60(n1046) does not suppress the Egl defect of lin-25(n545ts). This latter result suggests that let -60 may not be involved in the later event that requires lin-25. Similar observations have been made for $m p k$ 1/sur-1 (Wu and Han 1994). One possible explanation for inability of activated let-60 ras to suppress the Egl phenotypes caused by hypomorphic alleles of $l i n-25$ and $m p k-1 /$ sur-1, therefore, is that in some later signaling event the receptor transduces a signal via a worm homolog (not yet identified) of MEKK/STE11 (for review, see Mordret 1994) to mpk-1/sur-1 and thence to lin-25.

The egg-laying defect displays partial maternal rescue. For example, $\sim 7 \%$ of animals of the genotype lin-25(e1446) derived from an hermaphrodite of the genotype lin-25(e1446)/ + are able to lay some eggs and larvae before turning into bags of worms.

Extra VPCs We note that the production of seven pseudovulvae associated with $2^{\circ}$ lineages (Fig. 2 ) is evidence that the extra VPCs observed in lin-25 mutants (Ferguson et al. 1987) are indeed VPCs.

\section{Germ-line transformation experiments}

Microinjection of DNA into the germ line of C. elegans hermaphrodites was performed essentially as described by Mello et al. (1991). For lin-25 transformation rescue experiments, lin25(n545ts) hermaphrodites (raised at $15^{\circ} \mathrm{C}$ ) were injected with the plasmid pRF4 along with plasmid, cosmid, or $\lambda$ phage DNA potentially harboring the lin-25 gene. pRF4 encodes a dominant alelle of the rol-6 gene, su1006, which confers a Rol phenotype on transformed animals (Mello et al. 1991). Injected animals were placed on separate plates at $25^{\circ} \mathrm{C}$ and allowed to self-fertilize. Their progeny were allowed to develop at this temperature, which is nonpermissive for the n545ts mutation. $F_{1}$ Roller animals were placed on separate plates and scored for their ability to lay eggs and for the absence of bloating, a phenotype characteristic of egg-laying defective hermaphrodites (Trent et al. 1983). pRF4 plasmid DNA, purified by $\mathrm{CsCl}$ equilibrium density gradient centrifugation, was injected at a concentration of $50 \mu \mathrm{g} / \mathrm{ml}$. Test plasmid, cosmid, or $\lambda$ phage DNA was injected at a concentration of $\sim 15 \mu \mathrm{g} / \mathrm{ml}$. When the plasmid pSP2 was injected under these conditions, 82 of $100 \mathrm{~F}_{1}$ Roller animals were non-Egl. From these 100 worms, seven transformed lines were generated in which a significant proportion of the animals at each generation had inherited the extrachromosomal array formed from the injected plasmids. For all seven lines the majority of animals displaying the Rol phenotype were non-Egl. Injection of pSP2 into wild-type animals, even at high concentrations $(100 \mu \mathrm{g} / \mathrm{ml})$ did not cause the transformants to express any clearly visible phenotypes and did not reduce the number of transformants.

\section{Manipulation of recombinant DNA}

All standard molecular biological procedures were performed by use of methods described by Sambrook et al. (1989). DNA sequence was determined by use of the dideoxy chain termination method (Sanger et al. 1977) with reagents supplied by U.S. Biochemical. Except where otherwise noted, all sequencing reactions were performed with single-stranded DNA templates. To sequence lin-25 genomic DNA, conveniently sized pieces of the $10.1-\mathrm{kb}$ rescuing fragment were subcloned into the pBSII $\mathrm{KS} \mid-1$ $+\mid$ vectors and the resulting constructs used to generate nested sets of deletions by use of ExollI and S1 nucleases (Erase-a-base system, Promega). The sequence around sites used to generate subclones was confirmed by use of other clones. Sequence was analyzed with computer programs written by Staden (1986) and by the GCG (v. 7) (Devereux et al. 1984). Some programs from the MacVector software package (IBI) were also used.

\section{cDNA cloning}

Two Sall fragments, 1.6 and $1.85 \mathrm{~kb}$ in size, isolated from the plasmid pSP2 (Fig. 3C) were used to screen a C. elegans cDNA library kindly supplied by R. Barstead and R. Waterston (1989). Eight positive plaques were identified in a screen of $\sim 400,000$ phage clones. None of these cDNAs appeared to be full length: The largest, SNY4, was only $1.9 \mathrm{~kb}$. Restriction mapping, however, indicated that the two longest clones, SNY2 and SNY4, only partially overlapped. We sequenced all of one strand (the sense strand) of SNY2. This clone starts at position 661 in the lin-25 cDNA sequence (Fig. 5) and ends at position 2144. The clone resulted from oligo(dT) annealing to a polyadenine stretch in the middle of the lin-25 mRNA. We also sequenced most of one strand (the antisense strand) of SNY4. The sequence generated from this clone starts at position 2043 (Fig. 5) and ends (at one end of the clone) at position 3566 . This clone extends toward the $3^{\prime}$ end of the gene, past the translation stop codon at position 3571 , but does not contain a poly(A) tail. We therefore sequenced the ends of a number of other clones (by use of double-stranded DNA templates). SNY11 was found to contain a poly(A) tail, starting at position 3569, 16 nucleotides $3^{\prime}$ of a potential polyadenylation signal sequence, TATAAA. To isolate clones extending further $5^{\prime}$ we generated a probe from the $5^{\prime}$ end of SNY2 (position 661 to the EcoRI site at position 1553, 
Fig. 5) and used this to screen a cDNA library kindly supplied by J. Ahringer and J. Kimble (University of Wisconsin, Madison). Two, apparently identical, clones were isolated from a screen of $\sim 400,000$ phage clones. A 3.6-kb EcoRI fragment isolated from one clone, SNY16, was inserted into pBSII KS( + ) (Stratagene), and a section of one strand was sequenced. Analysis of the sequence revealed that the insert in SNY16 had arisen from the fusion of two different, unrelated cDNAs. The lin-25 sequence generated from SNY16 extends from position 245 to position 680 (Fig. 5).

\section{Reverse transcription-PCR}

A 72-bp single-stranded DNA primer (complementary to the lin-25 CDNA between positions 349 and 420 ) was labeled at the $5^{\prime}$ end and hybridized to $5 \mu \mathrm{g}$ of poly $(\mathrm{A})^{+}$RNA isolated from a mixed population of worms. Primer extension was performed under standard conditions by use of avian reverse transcriptase (Stratagene). One-fourth of the primer-extension reaction was analyzed by polyacrylamide gel electrophoresis under denaturing conditions to determine the size of the primer extended product $(s)$. One-eighth of the remainder was used as a template for PCR (30 cycles). One primer $15^{\prime}$-GTGGATGACTTGATTCCAGG-3') was complementary to a section of the lin-25 cDNA between positions 294 and 312 (Fig. 5) and the other had the sequence of the SL1 C. elegans splice leader RNA (5'-GGTTTAATTACCCAAGTTTGA-3') (Krause and Hirsh 1987). Southern blot analysis of the PCR products indicated that three different lin-25-related sequences $(\sim 120,230$, and 320 bp in size, respectively) had been amplified. Sections of a low-meltingpoint gel containing each of these three products were excised, and a small amount used as a template for a second round of PCR (30 cycles). In these reactions, one primer (5'-CGACAAGTTTGAGAAGATG $\left.\mathrm{C}_{-}-3^{\prime}\right)$ was complementary to a section of the lin-25 cDNA between positions 258 and 278 , and the other was the SL1 primer as before. Each reaction generated one band clearly visible on an ethidium bromide-stained agarose gel. All three fragments were cloned into pBSII KS|+ | (Stratagene) and sequenced. Analysis of the sequences revealed that the PCR products resulted from trans-splicing of the SL1 splice leader to the $5^{\prime}$ ends of exons 1, 2, and 3, respectively. Similar experiments with a primer that has the sequence of the SI2 splice leader $\left(5^{\prime}\right.$-GGTTTTAACCCAGTTACTCAAG-3') (Huang and Hirsh 1989/ showed that SL2 is also spliced to cach of the first three exons. However, it is possible that the SL2 products are extremely rare and were detected only because of the sensitivity of PCR after 60 cycles of amplification.

\section{Acknowledgments}

We thank K. Edwards, P. Stemberg, S. Clark, H.R. Horvitz, and M. Han for sharing unpublished results on Iin-25 both prior to the start of this study and during its progress. We are very grateful to S. Clark, H. Chamberlin, K. Edwards, D. Eisenmann, M. Han, H.R. Horvitz, S. Kim, and P. Sternberg for generously sending us their lin-25 alleles. We are indebted to A. Telfer and D. Stinchcomb for congenic strains (DT125 and DT127) and to G. Freyd and H.R. Horvitz for MT5798, which carries the lin$11:$ lacZ fusion. We thank A. Coulson and R. Shownkeen for fingerprinting $\lambda$ phage DNA and for sending us cosmids; $R$. Barstead, R. Waterston, J. Ahringer, and J. Kimble for cDNA libraries and the Caenorhabditis Genetics Center /which is funded by the National Institutes of Health National Center for Research Resources) for strains. We are very grateful to G. Beitel and H.R. Horvitz for sharing information concerning lin-1 and for allowing us to cite unpublished work. We thank E. Lambie for examining the germ lines of lin-25 mutant hermaphrodites. We gratefully acknowledge G. Beitel, B. Bucher, G. Kao, M. Sundaram, and J. Yochem for enlightening discussions and G. Beitel, J. Albert Hubbard, K. Fitzgerald, B. Grant, G. Kao, D. Levitan, and A. Tomlinson for comments on the manuscript. This work was supported by an Imperial Cancer Research Fund Travel Fellowship to S.T. and by a grant from the American Cancer Society (DB-35). I.G. is an Associate Investigator of the Howard Hughes Medical Institute.

The publication costs of this article were defrayed in part by payment of page charges. This article must therefore be hereby marked "advertisement" in accordance with 18 USC section 1734 solely to indicate this fact.

\section{Note added in proof}

The nucleotide sequence data reported in this paper have been deposited to the GenBank data library under accession no. U20168.

\section{References}

Ahnn, J. and A. Fire. 1994. A screen for genetic loci required for body wall muscle development during embryogenesis in Caenorhabditis elegans. Genetics 137: 483-498.

Altschul, S.F., W. Gish, W. Miller, E.W. Myers, and D.J. Lipman. 1990. Basic local alignment search tool. I. Mol. Biol. 215: 403-410.

Ambrosio, L., A.P. Mahowald, and N. Perrimon. 1989. Requirement of the Drosophila raf homologue for torso function. Nature 342: 288-291.

Aroian, R.V., M. Koga, J.E. Mendel, Y. Ohshima, and P.W. Sternberg. 1990. The let-23 gene necessary for Caenorhabditis elegans vulval induction encodes a tyrosine kinase of the EGF receptor subfamily. Nature 348: 693-699.

Barstead, R.J., and R.H. Waterston. 1989. The basal component of the nematode dense-body is vinculin. 1 . Biol. Chem. 264: 10177-10185.

Beitel, G.J., S.G. Clark, and H.R. Horvitz. 1990. Caenorhabditis elegans ras gene let-60 acts as a switch in the pathway of vulval induction. Nature 348: 503-509.

Birnstiel, M.L., M. Busslinger, and K. Strub. 1985. Transcription termination and $3^{\prime}$ processing: The end is in site Cell 41: 349-359.

Boulikas, T. 1993. Nuclear localization signals (NLS). Crit. Rev. Euk. Gene Exp. 3: 193-227.

Brand, A.H. and N. Perrimon. 1994. Raf acts downstream of the EGF receptor to determine dorsoventral polarity during Drosophila oogenesis. Genes \& Dev. 8: 629-639.

Brenner, S. 1974. The genetics of Caenorhabditis elegans. Genetics 77: 71-94.

Broach, J.R. and R.J. Deschenes. 1990. The function of RAS genes in Saccharomyces cerevisiae. Adv. Can. Res. 54: 79138.

Brunner, D., N. Oellers, J. Szabad, W.H. Biggs III, S.L. Zipursky, and E. Hafen. 1994a. A gain-of-function mutation in Drosophila MAP kinase activates multiple receptor tyrosine kinase signaling pathways. Cell 76: 875-888.

Brunner, D., K. Ducker, N. Oellers, E. Hafen, H. Scholz, and C. Klambt. 1994b. The ETS domain protein Pointed-P2 is a target of MAP kinase in the sevenless signal transduction pathway. Nature 370: 386-389.

Chang, F. and I. Herskowitz. 1992. Phosphorylation of FARl in response to $\alpha$-factor: A possible requirement for cell-cycle arrest. Mol. Biol. Cell 3: 445-450. 
Chou, S.-Y., V. Baichwal, and J.E. Ferrel. 1992. Inhibition of c-jun DNA binding by mitogen activated protein kinase. Mol. Biol. Cell 3: 1117-1130.

Clark, S.G., M.J. Stern, and H.R. Horvitz. 1992. C. elegans cellsignalling gene sem -5 encodes a protein with $\mathrm{SH} 2$ and $\mathrm{SH} 3$ domains. Nature 356: 340-344.

Clark, S.G., X. Lu, H.R. Horvitz. 1994. The Caenorhabditis elegans locus lin-15, a negative regulator of a tyrosine kinase signalling pathway, encodes two different proteins. Genetics 137: 987-997.

Clark-Lewis, I., I.S. Sanghera, and S.L. Pelech. 1991. Definition of a consensus sequence for peptide substrate recognition by $\mathrm{p} 44^{\mathrm{mpk}}$, the meiosis-activated basic protein kinase. J. Biol. Chem. 266: 15180-15184.

Coulson, A., J. Sulston, S. Brenner, and J. Karn. 1986. Toward a physical map of the genome of the nematode Caenorhabditis elegans. Proc. Natl. Acad. Sci. 83: 7821-7825.

Coulson, A., R. Waterston, J. Kiff, J. Sulston, and Y. Kohara. 1988. Genome linking with yeast artificial chromosomes. Nature 235: 184-186.

Crews, C.M., A. Alessandrini, and R.L. Erikson. 1992. The primary structure of MEK, a protein kinase that phosphorylates the ERK gene product. Science 258: $478-480$

Devereux, J., P. Haeberli, and O. Smithies. 1984. A comprehensive set of sequence analysis programs for the VAX. Nucleic Acids Res. 12: 387-395.

Ferguson, E.L. and H.R. Horvitz. 1985. Identification and characterization of 22 genes that affect the vulval cell lineages of the nematode Caenorhabditis elegans. Genetics 110: 17-72.

Ferguson, E.L., P.W. Sternberg, and H.R. Horvitz. 1987. A genetic pathway for the specification of the vulval lineages of Caenorhabditis elegans. Nature 326: 259-267.

Freyd, G., S.K. Kim, and H.R. Horvitz. 1990. Novel cysteine-rich motif and homeodomain in the product of the Caenorhab. ditis elegans cell lineage gene lin-11. Nature 344: 876-879.

Fukui, Y., Y. Kozasa, T. Takeda, and M. Yamamoto. 1986. Role of ras homolog in the life cycle of Schizosaccharomyces pombe. Cell 44: 329-336.

Gille, H., A.D. Sharrocks, and P.E. Shaw. 1992. Phosphorylation of transcription factor $\mathrm{p} 62^{\mathrm{TCF}}$ by MAP kinase stimulates ternary complex formation at $c$-fos promoter. Nature 358: 414 417.

Ginty, D.D., A. Bonni, and M.E. Greenberg. 1994. Nerve growth factor activates a Ras-dependent protein kinase that stimulates $c$-fos transcription via phosphorylation of CREB. Cell 77: 713-725.

Gonzalez, F.A., D.L. Raden, and R.J. Davis. 1991. Identification of substrate recognition determinants for human ERK1 and ERK2 protein kinases. J. Biol. Chem. 33: 22159-22163.

Greenwald, I.S., P.W. Sternberg, and H.R. Horvitz. 1983. The lin-12 locus specifies cell fates in Caenorhabditis elegans. Cell 34: 435-444.

Han, M. and P.W. Sternberg. 1990. let-60, a gene that specifies cell fates during C. elegans vulval induction, encodes a ras protein. Cell 63: 921-931.

Han, M., R.V. Aroian, and P.W. Sternberg. 1990. The let-60 locus controls the switch between vulval and nonvulval cell fates in Caenorhabditis elegans. Genetics 126: 899-913.

Han, M., A. Golden, Y. Han, and P.W. Sternberg. 1993. C. elegans lin-45 raf gene participates in let-60 ras-stimulated vulval differentiation. Nature 363: 133-140.

Herman, R.K. and E.M. Hedgecock. 1990. Limitation of the size of the vulval primordium of Caenorhabditis elegans by lin15 expression in the surrounding hypodermis. Nature 348: $169-171$.

Hill, R.J. and P.W. Sternberg. 1992. The gene lin-3 encodes an inductive signal for vulval development in C. elegans. $\mathrm{Na}$ ture 358: 470-476.

Horvitz, H.R. and J.E. Sulston. 1980. Isolation and characterization of cell-lineage mutants of the nematode Caenorhabditis elegans. Genetics 96: 435-454.

Huang, X.-Y. and D. Hirsh. 1989. A second trans-spliced leader RNA sequence in the nematode Caenorhabditis elegans. Proc. Natl. Acad. Sci. 86: 8640-8644.

Huang, L.S., P. Tzou, and P.W. Sternberg. 1994. The lin-15 locus encodes two negative regulators of Caenorhabditis elegans vulval development. Mol. Biol. Cell 5: 395-411.

Hunter, C.P. and W.B. Wood. 1992. Evidence from mosaic analysis of the masculinizing gene her-1 for cell interactions in C. elegans sex determination. Nature 355: 551-555.

Khosravi, R. and C.J. Der. 1994. The Ras signal transduction pathway. Cancer Metastasis Rev. 13: 67-89.

Kimble, J. 1981. Alteration in cell lineage following laser ablation of cells in the somatic gonad of Caenorhabditis elegans. Dev. Biol. 87: 286-300.

Kozak, M. 1986. Point mutations define a sequence flanking the AUG initiator codon that modulates translation by eukaryotic ribosomes. Cell 44: 283-292.

Krause, M. and D. Hirsh. 1987. A trans-spliced leader sequence on actin mRNA in C. elegans. Cell 49: 753-761.

Kurian, J. 1993. The pheromone response pathway in Saccharomyces cerevisiae. Annu. Rev. Genet. 27: 147-179.

Kyriakis, J.M., H. App, X. Zhang, P. Banerjee, D.L. Brautigan, U.R. Rapp, and J. Avruch. 1992. Raf-1 activates MAP kinasekinase. Nature 358: 417-420.

Lackner, M.R., K. Kornfeld, L.M. Miller, H.R. Horvitz, and S.K. Kim. 1994. A MAP kinase homologue, $m p k-1$, is involved in ras-mediated induction of vulval cell fates in Caenorhabditis elegans. Genes \& Dev. 8: 160-173.

Lai, Z. and G.M. Rubin. 1992. Negative control of photoreceptor development in Drosophila by the product of the yan gene, an ets domain protein. Cell 70: 609-620.

Leevers, S.J., H.F. Paterson, and C.J. Marshall. 1994. Requirement for Ras in Raf activation is overcome by targeting Raf to the membrane. Nature 369: 411-414.

Lu, X., T.-B. Chou, N. Gupta Williams, T. Roberts, and N. Perrimon. 1993. Control of cell fate determination by $\mathrm{p} 21^{\text {ras } /}$ Rasl, an essential component of torso signaling in Drosophila. Genes \& Dev. 7: 621-632.

Mango, S.E., E.M. Maine, and J. Kimble. 1991. Carboxy-terminal truncation activates glp-1 protein to specify vulval fates in Caenorhabditis elegans. Nature 352: 811-815.

Marais, R., J. Wynne, and R. Treisman. 1993. The SRF accessory protein Elk-l contains a growth factor-regulated transcriptional activation domain. Cell 73: 381-393.

Mello, C.C., J.M. Kramer, D. Stinchcomb, and V. Ambros. 1991. Efficient gene transfer in C. elegans: Extrachromosomal maintenance and integration of transforming sequences. EMBO I. 10: 3959-3970.

Miller, L.M., M.E. Gallegos, B.A. Morisseau, and S.K. Kim. 1993. lin-31, a Caenorhabditis elegans HNF-3/fork head transcription factor homolog, specifies three alternative fates in vulval development. Genes \& Dev. 7: 933-947.

Mordret, G. 1993. MAP kinase kinase: A node connecting multiple pathways. Biol. Cell 79: 193-207.

Nigon, V. 1949. Les modalities do la reproduction et le determinism du sex chez quelques Nematodes libres. Ann. Sci. Nat. Zool. 11: 1-132.

Ogiso, Y., T. Yokoyama, H. Watari, T. Shih, and N. Kuzumaki. 1993. Resistance of NIH3T3 cells to $v$-fes transformation induced by a dominant H-ras mutant. Exp. Cell Res. 208: $415-421$. 
O'Neill, E.M., I. Rebay, R. Tjian, and G. Rubin. 1994. The activities of two Ets-related transcription factors required for Drosophila eye development are modulated by the Ras/ MAPK pathway. Cell 78: 137-147.

Pearson, W.R. and D.J. Lipman. 1988. Improved tools for biological sequence comparison. Proc. Natl. Acad. Sci. 85: 2444-2448.

Peter, M., A. Gartner, J. Horecka, G. Ammerer, and I. Herskowitz. 1993. FARl links the signal transduction pathway to the cell cycle machinery in yeast. Cell 73: 747-760.

Ray, L.B. and T.W. Sturgill. 1987. Rapid stimulation by insulin of a serine/threonine kinase in 3T3-L1 adipocytes that phosphorylates microtubule-associated protein 2 in vitro. Proc. Natl. Acad. Sci. 84: 1502-1506.

Sambrook, J., E.F. Fritsch, and T. Maniatis. 1989. Molecular cloning: A laboratory manual, 2nd ed. Cold Spring Harbor Laboratory Press, Cold Spring Harbor, New York.

Sanger, F.S., S. Nicklen, and A.R. Coulson. 1977. DNA sequencing with chain terminating inhibitors. Proc. Natl. Acad. Sci. 74: 5463-5437.

Simon, M.A., D.D.L. Bowtell, G.S. Dodson, T.D. Laverty, and G.M. Rubin. 1991. Rasl and a putative guanine nucleotide exchange factor perform crucial steps in signaling by the sevenless protein tyrosine kinase. Cell 67: 701-716.

Song, O., J. Dolan, Y. Yuan, and S. Fields. 1991. Pheromonedependent phosphorylation of the yeast STE12 protein correlates with transcriptional activation. Genes \& Dev. 5: 741-750.

Staden, R. 1986. The current status and portability of our sequence handling software. Nucleic Acids Res. 14: 217.

Sternberg, P.W. 1988. Lateral inhibition during vulval induction in Caenorhabditis elegans. Nature 335: 551-554.

Sternberg, P.W. and H.R. Horvitz. 1986. Pattern formation during vulval development in C. elegans. Cell 44: 761-772.

1989. The combined action of two intercellular signaling pathways specifies three cell fates during vulval induction in C. elegans. Cell 58: 679-693.

Stokoe, D., D.G. Campbell, S. Nakielny, H. Hidaka, S.M. Leevers, C. Marshall, and P. Cohen. 1992. MAPKAP kinase2 ; a novel protein kinase activated by mitogen-activated $\mathrm{ki}$ nase. EMBO /. 11: 3985-3994.

Stokoe, D., S.G. Macdonald, K. Cadwallader, M. Symons, J.F. Hancock. 1994. Activation of Raf as a result of recruitment to the plasma membrane. Science 264: 1463-1467.

Sturgill, T.W., L.B. Ray, E. Erikson, and J.L. Maller. 1988. Insulin-stimulated MAP-2 kinase phosphorylates and activates ribosomal protein S6 kinase II. Nature 334: 715-718.

Sulston, J. and H.R. Horvitz. 1977. Postembryonic lineages of the nematode Caenorhabditis elegans. Dev. Biol. 56: 110156.

Sulston, J.E. 1976. Postembryonic development in the ventral cord of Caenorhabditis elegans. Phil. Trans. R. Soc. Lond. $B$ 275: 287-297.

Sulston, J.E and H.R. Horvitz. 1981. Abnormal cell lineages in mutants of the nematode Caenorhabditis elegans. Dev. Biol. 82: $41-55$.

Sulston, J.E. and J.G. White. 1980. Regulation and cell autonomy during postembryonic development of Caenorhabditis elegans. Dev. Biol. 78: 577-597.

Thomas, S.M., M. Demarco, G. D'Arcangelo, S. Halegoua, and J.S. Brugge. 1992. Ras is essential for nerve growth factor-and phorbol ester-induced tyrosine phosphorylation of MAP kinases. Cell 68: 1031-1040.

Treisman, R. and G. Ammerer. 1992. The SRF and MCM1 transcription factors. Curr. Opin. Genet. Dev. 2: 221-226.

Trent, C., N. Tsung, and H.R. Horvitz. 1983. Egg-laying defec- tive mutants of the nematode Caenorhabditis elegans. Genetics 104: 619-647.

Tsuda, L., Y.H. Inoue, M.-A. Yoo, M. Mizuno, M. Hata, Y.-M. Lim, T. Adachi-Yamada, and Y. Nishida. 1993. A protein kinase similar to MAP kinase activator acts downstream of the raf kinase in Drosophila. Cell 72: 407-414.

Van Aelst, L., M. Barr, S. Marcus, A. Polverino, and M. Wigler. 1993. Complex formation between RAS and RAF and other protein kinases. Proc. Natl. Acad. Sci. 90: 6213-6217.

Vojtek, A.B., S.M. Hollenberg, and J.A. Cooper. 1993. Mammalian Ras interacts directly with the serine/threonine kinase Raf. Cell 74: 205-214.

Warne, P.H., P. Rodriguez Viciana, and J. Downward. 1993. Direct interaction of Ras and the amino-terminal region of Raf- 1 in vitro. Nature 364: 352-355.

Waterston, R.H. 1981. A second informational suppressor, sup-7 $\mathrm{X}$, in C. elegans. Genetics 97: 307-325.

Wills, N., R.F. Gesteland, J. Karn, L. Barnett, S. Bolten, and R.H. Waterston. 1983. The genes sup-7 X and sup-5 III of C. elegans suppress amber nonsense mutations via altered transfer RNA. Cell 33: 575-583.

Wood, K.W., C. Sarecki, T.M. Roberts, and J. Blenis. 1992. ras mediates nerve growth factor receptor modulation of three signal-transducing protein kinases: MAP kinase, raf and RSK. Cell 68: 1041-1050.

Wu, Y. and M. Han. 1994. Suppression of activated Let-60 Ras defines a role of Caenorhabditis elegans sur-1 MAP kinase in vulval differentiation. Genes \& Dev. 8: 147-159.

Zhang, X.-F., J. Settleman, J.M. Kyriakis, E. Takeuchi-suzuki, and S.J. Elledge. 1993. Normal and oncogenic p21 ras proteins bind to the amino-terminal regulatory domain of c-Raf1. Nature 364: 308-313. 


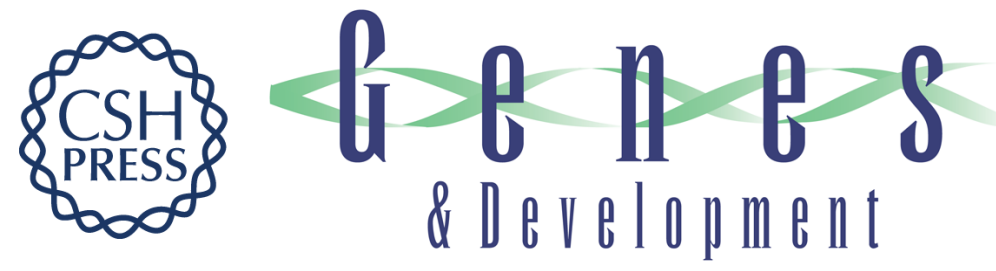

\section{lin-25, a gene required for vulval induction in Caenorhabditis elegans.}

S Tuck and I Greenwald

Genes Dev. 1995, 9:

Access the most recent version at doi:10.1101/gad.9.3.341

References This article cites 86 articles, 27 of which can be accessed free at: http://genesdev.cshlp.org/content/9/3/341.full.html\#ref-list-1

License

Email Alerting Receive free email alerts when new articles cite this article - sign up in the box at the top Service right corner of the article or click here.

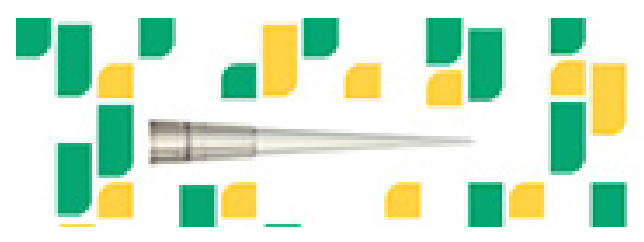

Focused on your science.

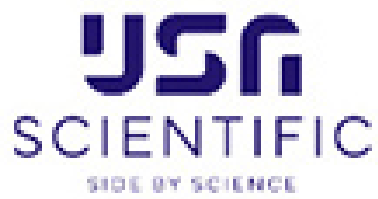

Copyright (c) Cold Spring Harbor Laboratory Press 